\title{
Acompañamiento a emprendedores en la Universidad EAN: Del modelo IN3 a EAN Impacta -2011-2016-
}

\section{Resumen}

Desde su fundación, la Universidad EAN es pionera en ofrecer educación enfocada a fortalecer competencias emprendedoras. Por este motivo, es siempre un desafío diseñar programas enfocados en potenciar emprendedores. Este artículo enmarca el acompañamiento emprendedor dentro de unas tendencias globales y necesarias en el ecosistema colombiano, tales como: el pensamiento en diseño aplicado en los negocios, el emprendimiento ágil, los programas de aceleración, y la innovación a partir de la investigación. Lo anterior, se ha convertido en buenas prácticas para la incubación de empresas desde las universidades (Bhatli, 2014). De esta forma, se analiza la evolución, procesos y resultados de los programas de acompañamiento desarrollados en la Universidad EAN: Modelo IN3 -Incuba-Innova-Integra-, el Punto de Atención al Emprendedor (PAE) y su Fondo de Capital Semilla; y finalmente, el programa actual llamado EAN Impacta.

\section{Palabras clave}

Emprendimiento, incubación empresarial, unidades de emprendimiento, modelos de atención emprendedora, Universidad EAN.

\section{Jairo Alonso Orozco Triana}

Magíster en Gestión de la Innovación, Universidad Tecnológica de Bolívar; especialista en Finanzas, Universidad EAFIT; profesor asociado del Instituto para el Emprendimiento Sostenible, Universidad EAN.

\section{León Darío Parra Bernal}

PhD. en Economía, Universidad Autónoma de México; magíster en Population Studies, Facultad Latinoamericana de Ciencias Sociales; profesor asociado del Instituto para el Emprendimiento Sostenible, Universidad EAN.

\section{Catalina Lucía Ruíz Arias}

Magíster en Diseño Estratégico de Producto, Delft University of Technology -Holanda-; profesor asociado del Instituto para el Emprendimiento Sostenible, Universidad EAN.

\section{Francisco Javier Matíz Bulla}

PhD (c) y DEA en Gestión de la Nueva Economía, Universidad de las Palmas de Gran Canaria -España-; magíster en Gestión de Organizaciones, Universidad de Québec -Canadá-; especialista en Sicología del Consumidor, Universidad Konrad Lorenz; director del Instituto para el Emprendimiento Sostenible, Universidad EAN. 


\section{Tutoring to Entrepreneurs at Universidad EAN: from the IN3 Model to EAN Impact -2011-2016-}

Abstrac. Since its foundation, Universidad EAN has been a pioneer in offering formation and training to foster entrepreneurial competencies; for this reason, it has always been a permanent challenge to design programs focusing on the formation of entrepreneurs. This article describes the entrepreneurs' tutoring and follow- up made following necessary global trends in Colombian ecosystems, such as applied design thought on business, agile entrepreneurship, accelerating programs and innovation resulting from research, which have become the best practices of company incubation for universities (Bhatli, 2014). From this start, we analysed the evolution, processes and outcomes of Universidad EAN tutoring and follow- up programs: IN3 Model (Incubate- Innovate- Integrate), the Entrepreneur Assistance Office ("PAE" in Spanish) and the Seed Capital Funding, as well as the current program named EAN Impact.

Key words. Entrepreneurship, company incubation, entrepreneurship offices, entrepreneurial assistance models, Universidad EAN.

\section{Accompagnements des entrepreneurs de I'Université EAN: du Modèle IN3 à EAN Impact -2011-2016-}

Resumé. L'Université EAN est, depuis sa fondation, pionnière en Colombie en termes d'offre de cursus éducatifs dédiés à l'amélioration des compétences entrepreneuriales cherchant de manière permanente à créer des programmes destinés à renforcer les compétences des entrepreneurs. Cet article offre une synthèse du type d'accompagnement entrepreneurial offert par l'Université EAN et les tendances globales de l'écosystème colombien tels que : la création entrepreneuriale, l'entrepreneuriat agile, les programmes d'amélioration et d'innovation fondés sur la recherche universitaire se convertissant en pratiques d'incubation d'entreprises (Bhatli, 2014). Cet article analysera l'évolution, les processus et les résultats des programmes d'accompagnement mis en place par I'Université EAN : le Modèle IN3 (Incubation-Innovation-Intégration), le Service d'Accompagnement à l'Entrepreneur (SAE) et son Fond de Capital Risque ainsi que le programme EAN Impact.

Mots clefs. Entrepreneuriat, incubation entrepreneuriale, unités entrepreneuriales, modèle d'accompagnement de l'entrepreneur, Université EAN.

\section{Acompanhamento a empreendedores na Universidade EAN: do modelo IN3 a EAN Impacta -2011-2016-}

Resumo. Desde sua fundação, a Universidade EAN é pioneira em oferecer educação enfocada ao fortalecimento de competências empreendedoras; por este motivo é sempre um desafio desenhar programas enfocados em potencializar empreendedores. Este artigo enquadra o acompanhamento empreendedor dentro de umas tendências globais e necessárias dentro do ecossistema colombiano, como: o pensamento em desenho aplicado nos negócios, o empreendimento ágil, os programas de aceleração e a inovação a partir da investigação, os quais têm se convertido em boas práticas para a incubação de empresas desde as universidades (Bhatli, 2014). A partir do anterior, analisa-se a evolução, os processos e os resultados dos programas de acompanhamento desenvolvidos na Universidade EAN: Modelo IN3 (Incuba-Inova-Integra), o Ponto de Atenção ao Empreendedor (PAE) e seu Fundo de Capital Semente, e, finalmente o programa atual chamado EAN Impacta. Estes programas realizam-se para oferecer aos empreendedores Eanistas elementos de apoio para levar à realidade suas inciativas empresarias. A análise começa desde 2011 com o modelo IN3 até o modelo atual EAN Impacta, fazendo ênfase em como o primeiro estabelece as bases para o desenvolvimento do modelo atual e como este se potencializa com as novas tendências globais em incubação empresarial

Palabras chave. Empreendimento, incubação empresarial, unidades de empreendimento, modelos de atenção empreendedora, Universidade EAN. 


\section{Introducción}

Tl proceso de creación y consolidación de Cempresas en etapas tempranas son retos urgentes que hay que superar para aportar al crecimiento y desarrollo económico de los países. Sobre esta temática existe una variada evidencia empírica que apunta a la relación positiva entre la creación de nuevas empresas

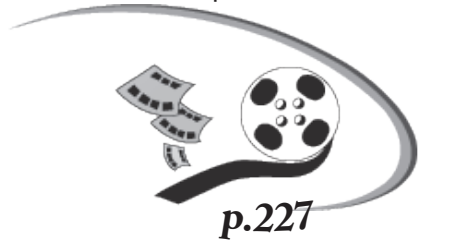

y el crecimiento económico de un país (Reynolds, 2000 y 2001). Sin embargo, todavía existen aspectos que requieren atención, como conocer los tipos de empresas que generan riqueza, los factores que intervienen en el éxito o fracaso empresarial y el tipo de apoyo que deben recibir los nuevos empresarios para sacar sus empresas adelante (Kantis, et. al., 2002).

Con base en estos aspectos, surge la necesidad de fortalecer la ejecución de programas enfocados al emprendimiento. Este fortalecimiento se genera a partir del análisis de la experiencia y de los resultados de estos programas, con el propósito de plantear acciones de mejora al proceso de gestión de acompañamiento emprendedor. De esta forma, se optimizan las empresas para que generen impactos positivos en el desarrollo económico. En este sentido, el presente artículo tiene como objetivo describir y analizar la evolución de los programas de la Universidad EAN de Colombia enfocados a la incubación de empresas en etapas tempranas y los resultados obtenidos desde el año 2011 al 2016, con el fin de que sirva como un caso de estudio en el marco de los programas de emprendimiento que se vienen desarrollando en América Latina.
La Universidad EAN es pionera en Colombia en incorporar, de forma transversal, el emprendimiento en su modelo educativo pues ha ido evolucionando su modelo de incubación en los últimos seis años; primero, con el programa Incuba-Innova-Integra (IN3), el programa Punto de Atención al Emprendedor (PAE) y actualmente con el programa EAN Impacta, con resultados factibles por evaluar para una retroalimentación del proceso de gestión empresarial de la Institución.

Con el fin de documentar esta experiencia enriquecedora, los siguientes apartados describirán las estrategias de apoyo a los emprendedores de la Universidad EAN, lo cual será información de relevancia con miras a resaltar las buenas prácticas y el proceso de aprendizaje de un modelo a otro, teniendo en cuenta las cambiantes tendencias del emprendimiento.

La estructura del artículo está compuesta por un marco conceptual que describe referencias relacionadas con la importancia del fomento al emprendimiento en países de Latinoamérica, incluido Colombia, y su impacto en el desarrollo económico. Seguidamente, se describen los programas de incubación empresarial en la Universidad EAN, sus metodologías, fases y resultados, y, sobre todo, las lecciones aprendidas de cada uno de esos programas, las cuales robustecieron la Institución para establecer el programa EAN Impacta utilizado actualmente. Al finalizar, se relacionan los aprendizajes generales y las perspectivas de la incubación empresarial desde la Universidad EAN. 


\section{Marco conceptual}

P ara profundizar en el tema de la incubación empresarial, se requiere contextualizar la temática de emprendimiento incluyendo las teorías que la fundamentan. En este sentido, a continuación se presentan los conceptos más importantes sobre el empresario y la cultura empresarial que se han abordado desde diferentes paradigmas, para con ello centrar el análisis en el proceso de gestión empresarial en el contexto colombiano y estudiar, como caso particular, la evolución de los programas de incubación de la Universidad EAN.

\subsection{El empresario, la cultura empresarial y el desarrollo económico}

Desde su mención por parte de Cantillon (1896), los conceptos de empresario y cultura empresarial han sido objeto de un estudio sistemático que ha llevado a su consolidación en la teoría económica de la firma (Perdomo, 2010).

Para poder comprender esta forma de ver la competencia y el mercado dentro de la teoría económica, en la cual está inmersa la cultura empresarial, se requiere de una total transformación en el enfoque económico que considera a las firmas como una caja negra en un agregado económico perfectamente racional, que es retroalimentado por las transacciones económicas y por los precios (Kantis et. al, 2004). En contraste, es factible ver dentro del agregado económico un conjunto de procesos para fabricar, vender y comprar, en el cual el empresario se apropia de un papel imprescindible para descubrir la necesidad, que en conjunción con los beneficios puros y la información de la oferta y la demanda del mercado, logra una función empresarial que es la esencia de la empresarialidad (Kirzner, 1998).

Según Foss y Klein (2004), el espíritu empresarial se ha convertido en una de las temáticas más desarrolladas en la economía moderna. Se pueden mencionar algunos autores como Hayek (1934), que aborda estos conceptos enfatizando en la creatividad empresarial; Mises (2001), que lo hace desde la noción de acción humana, y Kirzner (1998), quien claramente profundiza en el emprendedor como un innovador. Esta última característica del empresario es el pilar de su concepción dinámica y progresista; conceptos que fueron desarrollados plenamente por Schumpeter (1934) y que son considerados con bastante relevancia en la economía actual.

Dentro de las principales contribuciones de Schumpeter está la diferenciación entre crecimiento económico y desarrollo económico. Para este autor, el crecimiento supone adaptación, mientras que desarrollo implica un cambio desde adentro a través de la innovación creativa (Schumpeter,1934). La mencionada adaptación implica considerar factores como el crecimiento demográfico, con base en que las economías se adaptan naturalmente, mientras que la innovación se genera desde adentro y la génesis de este proceso innovador parte del empresario.

Esta función innovadora del emprendimiento, que parte del empresario, genera lo que se denomina destrucción creativa, entendida como el cambio novedoso de lo anterior o actual por algo nuevo que satisfaga de mejor manera las necesidades de los otros (Schumpeter, 1934). 
En el caso de Latinoamérica, el informe publicado por el Banco Mundial (Lederman et $a l, 2014)$ en una muestra de 55 países latinoamericanos, europeos y africanos con similares características, pone de manifiesto que el número de empresarios latinoamericanos per cápita es mayor que en los países de las otras regiones estudiadas.

La misma fuente describe que estos empresarios latinoamericanos se caracterizan, en gran medida, por la formalidad, pues existe un gran porcentaje de empresas que están registradas ante las autoridades respectivas. A pesar de lo anterior, el informe del Banco Mundial (Lederman et al, 2014) concluye que existe una problemática recurrente en las empresas latinoamericanas: la escasez de innovación. Agrega que existe una brecha sustancial y crónica en términos de innovación, entre Latinoamérica y el Caribe, y los países y regiones, comparables en términos de crecimiento económico. La brecha no solo se evidencia en el ámbito de la investigación y desarrollo (I+D) y las patentes, sino también en el contexto de la innovación de productos y procesos. Por otro lado, se presentan grandes diferencias a nivel intra e intersectorial, los cuales llevan a manifestar brechas tecnológicas que dificultan la generación de crecimiento dinámico en la región (Parra y Argote, 2013; Lederman et al., 2014).

Por su parte, Kantis et al. (2004), en un estudio publicado por el Banco Interamericano de Desarrollo (BID), proponen que para llevar a cabo un apoyo integral en el proceso de gestión del emprendimiento que desarrolle una cultura empresarial más innovadora, se deben estimular y motivar nuevos emprendimientos a través de la promoción de modelos de empresarios en el sistema educativo, como referentes, fomentar la innovación y la creatividad, estimular la conformación de redes de empresarios o equipos de emprendedores de alto desempeño, facilitar el periodo de gestación del nuevo emprendimiento, acompañar en el desarrollo del plan de negocio, así como facilitar la obtención y análisis de la información pertinente para la toma de decisiones de los emprendedores.

Claramente, en varios países de Latinoamérica, como Chile, México, Brasil, incluyendo Colombia, se vienen aplicando políticas del emprendimiento que evidencian el crecimiento del tejido productivo y la generación de empleo (Kantis, 2004). En Brasil, el trabajo realizado por el programa SOFTEX y su incubadora de empresas ha logrado permear el ámbito nacional y local a lo largo de los últimos diez años, posicionando a este país entre los primeros lugares de exportación de software. El programa contribuyó a la expansión de las exportaciones de software, que pasaron de un millón de dólares en 1990 a 100 millones en el año 2001. De igual forma, la Corporación para el Fomento de la Producción (Corfo), en Chile, ha impactado en el desarrollo de incubadoras de empresas chilenas de clase mundial, y varias de estas han sido reconocidas por University Business Incubator (UBI) como las más destacadas en Latinoamérica.

Los estudios realizados en el Banco Mundial y BID proponen políticas públicas orientadas al impulso de la capacidad emprendedora a través de una estrategia integral con una planificación a largo plazo de las iniciativas de promoción empresarial. En resumen, lo que el Banco Mundial, a través de este informe recomienda, es que estas iniciativas impulsen al empresario desde lo técnico, lo financiero y lo formativo, para desarrollar una cultura empresarial, y, en consecuencia, lleven al desarrollo económico. Indudablemente, las leyes y normativas como herramientas de política no van a ser suficientes para mejorar la innovación de las empresas en Latinoamérica (Lederman et al., 2014). 
Esta revisión permite concluir que parte de los principales retos de las políticas se relacionan con los aspectos estructurales más profundos del entorno favorable al emprendimiento innovador, que no solo incluyen las leyes y las instituciones, sino también las dotaciones de infraestructuras y la cantidad y calidad de capital humano, de las cuales forman parte las universidades, las incubadoras de empresas, los fondos de capital y otras instituciones creadas para brindar soporte al emprendedor. Es allí donde radica la importancia de conocer experiencias de actores latinoamericanos del ecosistema emprendedor, para que se conviertan en una base de conocimiento en la industria de soporte.

\subsection{La promoción de la cultura empresarial en Colombia}

El tema de emprendimiento en Colombia ha ido emergiendo paulatinamente desde 1990, momento en el cual se dictaron disposiciones, como la Ley 29, para el fomento de la investigación científica y el desarrollo tecnológico, y se otorgaron facultades extraordinarias. En 1996 ya se habían definido normas encaminadas a la racionalización del gasto público para fomentar la innovación en las empresas con la Ley 344. En el año 2000 se sancionó la Ley 590 de promoción del desarrollo de la micro, pequeña y mediana empresa colombiana, expedida en el Plan Nacional de Desarrollo 2006-2010. El 2002, por la Ley 789, se creó el Fondo Emprender con el objetivo de financiar iniciativas empresariales a estudiantes y profesionales de diferentes ámbitos y bajo determinadas características ${ }^{1}$.
Dentro de los planes nacionales de desarrollo de Colombia en los últimos 15 años, existe la Política Nacional de Emprendimiento y la Innovación a través del Ministerio de Industria y Comercio. Una de las instituciones creadas por el Estado es iNNpulsa, cuyo fin es promover o fomentar el emprendimiento con alto potencial de crecimiento. Propone estrategias de promoción de la cultura empresarial en el país, de manera integral, a través del fortalecimiento de las capacidades de incubación y aceleración de entidades de acompañamiento, impulsando el cambio de mentalidad de los emprendedores hacia el crecimiento y acompañarlos a financiar y potenciar sus iniciativas innovadoras.

Partiendo de la Política Nacional de Emprendimiento y la Innovación, uno de los programas con mayor impacto es el Fondo Emprender, entidad que apoya proyectos empresariales que integren los conocimientos adquiridos por los emprendedores en sus procesos de formación con el desarrollo de nuevas empresas, facilitando el acceso a capital semilla para la puesta en marcha de las nuevas unidades productivas. Para 2016, este Fondo ha impactado a 4900 empresas que generan alrededor de 17 mil empleos. En Medellín se creó Cultura E, un programa promovido y financiado por la Alcaldía de la misma localidad, que busca masificar la cultura del emprendimiento, el desarrollo de nuevas empresas que respon-dan a las necesidades del mercado y a las dinámicas de las cadenas productiva regionales con mayores potencialidades económicas, valiéndose de la capacidad de innovación de sus emprendedores. También, Manizales lanzó el programa Manizales + con el apoyo de Babson College, para potenciar los emprendimientos en la región; estos son ejemplos de las políticas públicas enfocadas al emprendimiento.

1 Las condiciones de beneficiarios del Fondo Emprender se establecen ampliamente en el Acuerdo 004 del 26 de marzo del 2009. 
Peña-Vinces et. al. (2010) concluyen que la promoción o fomento del emprendimiento por medio de incubadoras de empresas ha tenido un gran desarrollo en Colombia. Se concibe que la incubación realiza diversos procesos de acompañamiento o coaching, capacitación, asesoría técnica y financiera, como apoyo integral a los emprendedores. En Colombia, la creación de empresas a través de incubadoras y programas de emprendimiento, ha sido una estrategia ampliamente difundida como mecanismo de apoyo al sector empresarial. Varias incubadoras de Bogotá, Bucaramanga, Cali y Medellín son generadoras de empleos (PeñaVinces et. al., 2010; GEM, 2013). Igualmente, las universidades han asumido un rol clave en la educación enfocada a las competencias para la creación de empresas y se les exige cada vez más asumir los desafíos de la promoción del emprendimiento, sobre todo, el relacionado con la innovación a partir de los resultados de las investigaciones (ASCUN, MINCIT, 2014).

En este marco de operación, la Universidad EAN ha incorporado el tema del emprendimiento desde su fundación en 1968. Los objetivos de este proyecto educativo son ofrecer a las nuevas generaciones programas de formación integral y humanista, en el marco de las ciencias y técnicas administrativas, con énfasis en la formación de empresarios y en el fomento del espíritu empresarial, de tal manera que los egresados de estos programas puedan contribuir al desarrollo económico y social de los pueblos.

Cabe resaltar, que, para que este ejercicio de retroalimentación y aprendizaje de buenas prácticas sea constante, es de vital importancia la construcción de sistemas de información sobre emprendimiento, la recopilación de datos nacionales y locales, la realización de investigaciones comparadas y de evaluación de impacto, a partir de las cuales se logre diagnosticar la situación actual y modelar las expectativas a futuro. En este sentido, este artículo aporta, desde 2011, la experiencia de la Universidad EAN con relación a la incubación y al fomento de empresas innovadoras dentro de la comunidad, tomando como base sus programas IN3, PAE y EAN Impacta, que ponen de manifiesto el emprendimiento como parte de sus fundamentos estratégicos institucionales.

\section{Modelos de incubación en la EAN}

\section{I}

os programas de incubación desarrollados en la Universidad EAN han tenido, como objetivo principal, integrar el proceso formativo transversal en emprendimiento con un acompañamiento integral para potencializar las ideas de negocio de los estudiantes y egresados EANistas con componentes innovadores y ventajas competitivas en el mercado, siendo esta una acción concreta de proyección social hacia la comunidad de la Universidad. El primer programa de incubación se denominó programa IN3, que operó desde el año 2011 y tuvo como objetivo viabilizar las ideas, modelos y planes de negocio generados por los estudiantes y egresados, acompañándolos en el crecimiento de sus proyectos emprendedores (Parra y Argote, 2013).

Con la creación del Instituto de Emprendimiento, Liderazgo e Innovación (IDELI) en 2013, el IN3 se transformó en el PAE, que dió continuidad al modelo de incubación 
y aceleración, complementando el acompañamiento con un Fondo de Capital Semilla de la Universidad EAN, iniciativa que contó con presupuesto propio para el financiamiento de proyectos de emprendedores EANistas. Después de tres versiones de este Fondo -2013 al 2015-, con el desarrollo de un proyecto de fortalecimiento de capacidades de incubación y aceleración cofinanciado por iNNpulsaBancoldex, se incluyeron nuevas metodologías y buenas prácticas, y se estructuró el programa EAN Impacta, el cual se enfocó en el acompañamiento y fortalecimiento en aspectos técnicos, estratégicos y de habilidades blandas a iniciativas empresariales y sus emprendedores, parafacilitarlapuestaen marchadelasempresas, asegurar su sostenibilidad y aprovechar los servicios y acceso a capital en el ecosistema emprendedor en Colombia y Latinoamérica. A partir de la inclusión de la sostenibilidad en la política general de la Universidad EAN, el IDELI se transformó en un organismo que integra la sostenibilidad, la innovación, el liderazgo y el emprendimiento, cambiando su nombre a
Instituto para el Emprendimiento Sostenible. Dentro de su estructura organizacional se incorpora claramente el programa EAN Impacta, como proceso de extensión del Instituto.

En los siguientes apartes se describe la estructura del programa IN3, su proceso de evolución con la experiencia del Fondo de Capital Semilla de la Universidad EAN, así como el nuevo programa EAN Impacta resultante.

\subsection{Modelo de incubación extramuros IN3}

El IN3 buscaba materializar las ideas emprendedoras en planes de negocio susceptibles a ser incubados y transformados en empresas de base tecnológica, a través de tres áreas de operación subdivididas en seis fases de incubación, que permitieron gestionar y viabilizar los planes de negocio de los emprendedores (Figura 1).

Figura 1. Modelo de incubación extramuros IN3.

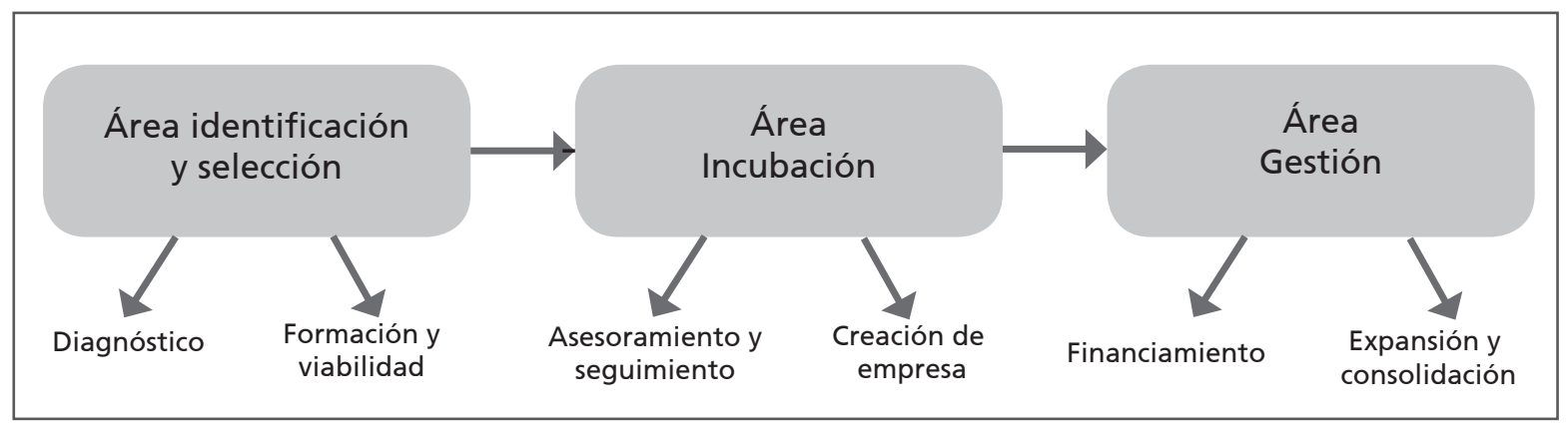

Fuente. Elaboración propia de los autores. 
El área de identificación y selección consistía en la inscripción y entrevista personal para la determinación de los requerimientos y necesidades de los planes de negocios inscritos. El área de incubación, por su parte establecía las estrategias de asesoramiento y seguimiento para la creación de la empresa mediante la verificación del cumplimiento de un plan estratégico empresarial plasmado objetivamente en un plan de negocios. El propósito del área de gestión era apoyar a los participantes en la búsqueda de nuevos mercados y de fuentes de financiamiento que apalancaran aquellos planes de negocios que estuvieran en una etapa de maduración avanzada para poder ser sostenibles a largo plazo. Este modelo se convirtió en la base de lo que hoy se conoce como EAN Impacta.

\subsection{1 Área de identificación y selección}

Esta área funcionaba como el proceso de la selección y priorización sistemática de las ideas o planes de negocio inscritos a determinada convocatoria de incubación. Como órgano de decisión se conformó un comité evaluador para analizar el nivel de madurez de la idea o proyecto. También, este comité identificaba el perfil del postulante y verificaba que la propuesta incorporara elementos innovadores, con el fin de seleccionarlo como candidato potencial para participar en IN3.

La captación de potenciales emprendedores se realizaba a través de convocatorias abiertas y trimestrales a lo largo del ciclo escolar, para dar un cubrimiento total a la comunidad universitaria, compuesta por estudiantes, colaboradores y egresados. La difusión de las convocatorias se realizaba a través de medios virtuales, con los docentes del área de emprendimiento en las unidades de estudio de creación de empresas e iniciativa empresarial, y a los finalistas del EAN Entrepreneurship Award, concurso que realiza anualmente la universidad.

El proceso de evaluación de cada propuesta se concretaba en una categorización de tres niveles: emprendedores nacientes, junior y senior. El tipo de emprendedor naciente tiene un plan de negocio que se encuentra en una fase incipiente de desarrollo, pero manifiesta potencial empresarial y elementos diferenciales en su propuesta de valor. Esta fase incipiente se consideró como un emprendedor con una idea clara sin tener definidos los elementos que la configuran en un plan de negocios. El tipo de emprendedor junior cuenta con un plan de negocios estructurado y con una planificación tendiente a alcanzar metas determinadas. El tipo de emprendedor senior cuenta con una empresa en marcha que posee aún una limitada experiencia en el mercado, pero que tiene potencial de expansión.

La filosofía de IN3 estaba basada en brindar apoyo técnico y financiero a la medida de los emprendimientos. En este sentido, a cada tipo de emprendimiento le correspondió una estrategia diferente de acompañamiento y asesoría. Para el nivel incipiente, se diseñó una asesoría directa e individual de dos horas quincenales con un docente; para el nivel intermedio, se planeó una de carácter mixto -directo y virtual-; y para el nivel avanzado, la asesoría se realizaba utilizando objetos de aprendizajes y ambientes virtuales a través de la plataforma blackboard, en la que se establecía la comunicación y atención del emprendedor y el programa (Parra y Argote, 2013). 


\subsection{2 Área de incubación}

El objetivo de esta área se centró en brindar un acompañamiento extramuros al emprendedor en el proceso de puesta en marcha de su empresa. Se estructuró con un coordinador para seguir el cumplimiento de los objetivos propuestos en el programa y con docentes asesores, como tutores, que daban apoyo y asesoría presencial a los emprendedores, con una regularidad e intensidad de dos horas cada quince días, durante aproximadamente tres meses.

El área de incubación se concentró principalmente en consolidar la fase de asesoría y seguimiento de las ideas o planes de negocio. El asesoramiento buscaba que el emprendedor formulara o mejorara su plan de negocios -aspectos operacionales, técnicos, de mercadeo y financieros- y pudiera plantear de manera óptima la propuesta de valor. Con el plan de negocios bien elaborado, el seguimiento consistió en el apoyo a la planificación del proceso de arranque de la empresa a través de un acompañamiento a los emprendedores.

\subsection{3 Área de gestión}

Esta área se dedicó, principalmente, a apoyar al emprendedor en la búsqueda de recursos financieros y comerciales para la creación y consolidación de las empresas incubadas. En este sentido, se trabajó identificando fuentes de financiamiento; además, vinculando al emprendedor a la red de contactos con inversionistas y clientes potenciales. En cuanto a su estructura organizacional, al igual que las dos áreas anteriores, se conformó con un coordinador de área, un equipo de docentes asesores y la red de aliados estratégicos, quienes contribuyeron al desarrollo y puesta en marcha de cada nuevo emprendimiento.
Dentro de las buenas prácticas se destacaban los encuentros empresariales para que los emprendedores y empresas en proceso de crecimiento y consolidación adquiriean más destrezas y habilidades, a través de cine foros, talleres, paneles empresariales, ruedas de negocios y tertulias, encuentros sectoriales que forman parte de los eventos regulares de la Universidad para el fomento del emprendimiento y en los cuales el programa IN3 participaba activamente con el grupo de emprendedores. Estas actividades eran gestionadas y organizadas en su mayoría por la Universidad, algunas veces subcontratando a terceros o vinculando a diversos actores del ecosistema.

Una de las actividades que se destacaron fue el intercambio de información y la conformación de redes de contactos con empresas a través de ruedas de negocio y de innovación. En estos espacios se contabilizaban las reuniones entre emprendedores, y aquellos que podían acceder a clientes potenciales a partir de una plataforma virtual destinada para tal efecto. Esta plataforma se planteó como un servicio temporal, prestado por un proveedor experto en el desarrollo de ruedas de negocios.

Como resultado del programa, los primeros indicadores en los semilleros incubados entre 2011 y 2012 mostraron una respuesta positiva de la comunidad EANista, teniendo en cuenta la experiencia previa de la Universidad en el trabajo con emprendedores. Las cifras del programa fueron 73 proyectos postulados, de los cuales el $50 \%$ lograron llegar a la fase de gestión de financiamientos; de estos, el $10 \%$ son empresas creadas o en funcionamiento que han accedido a financiamientos públicos o privados. 


\subsection{Lecciones aprendidas del programa IN3}

La experiencia de la Universidad EAN en los temas de emprendimiento y la red de contactos que ha consolidado a lo largo de los años son fundamentales para el establecimiento de procesos de acompañamiento a emprendedores.

La principal fortaleza de la Universidad EAN es la promoción de la cultura empresarial desde sus propios fundamentos estratégicos que impulsan la formación de profesionales con espíritu empresarial, lo cual indica el compromiso desde la alta dirección de la Institución y su apoyo a las actividades de fomento. La implementación de modelos de incubación es la continuación natural del modelo educativo de la Universidad, que busca fortalecer las competencias en los emprendedores en el uso de herramientas prácticas para la formulación de sus planes de negocio, como punto de partida estratégico hacia la consecución de fondos de capital y la puesta en marcha de la empresa.

El principal reto del programa IN3 se relacionó con el acceso a financiamiento. Para esto, se focalizaron las fortalezas y debilidades de los emprendedores y se lograron alianzas estratégicas. A partir de esta situación, se comenzaron a otorgar incentivos económicos por medio del Fondo de Capital Semilla de la Universidad EAN.

Otro aprendizaje fue la necesidad de fotalecer la sistematización de la información para facilitar el monitoreo, trazabilidad y evaluación de los procesos y fases de la incubación, y la generación de información para emprendedores e inversionistas con el fin de apoyar la toma de decisiones. Esta valiosa experiencia de IN3 se tuvo en cuenta para el rediseño del programa que derivó en EAN Impacta, el cual utiliza una plataforma en línea para llevar a cabo la trazabilidad de los emprendedores en todas las etapas del proceso de incubación.

Otro de los retos que enfrentó el programa fue la relación entre tutores y emprendedores, para fomentar el cumplimiento de sus compromisos y la orientación al logro. En un proceso de incubación es fundamental contar con los recursos humanos necesarios y acordes con la demanda, y con la capacidad requerida para el logro de las metas del proceso de incubación. Se necesitan mecanismos para fomentar el cumplimiento de los compromisos de todos los involucrados, en especial de los emprendedores, así como su persistencia para la puesta en marcha de las empresas y su sosenibilidad. En este sentido, con la creación del IDELI -actual Instituto para el Emprendimiento Sostenible-, se inició el proceso de estructurar un equipo de trabajo, gestionar recursos, y teniendo en cuenta la experiencia del IN3, aumentar la capacidad para brindar servicios de acompañamiento a los emprendedores.

El equipo de trabajo

planteado para mejorar el programa incluyó los perfiles relacionados con la gestión de

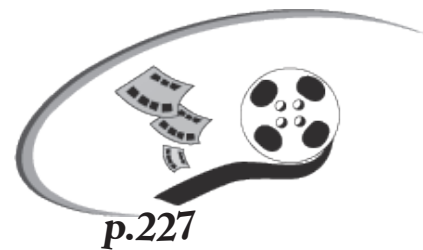
proyectos, el diseño, ingenierías, la consultoría y las ciencias empresariales, sobre todo, en la experiencia empresarial y en programas de acompañamiento emprendedor. Con el equipo de trabajo y nuevos procedimientos desarrollados a partir de un proceso de fortalecimiento en las capacidades de incubación, dentro de un programa con iNNpulsa, se buscó generar mayor compromiso de los emprendedores. 


\subsubsection{Del Programa IN3 al Programa EAN Impacta}

Desde el 2013, con la creación del Instituto de Emprendimiento, Liderazgo e Innovación (IDELI) -actual Instituto para el Emprendimiento Sostenible- y la implementación del Fondo de Capital Semilla de la Universidad EAN, los esfuerzos de acompañamiento del IN3 se transformaron en el PAE, desde el cual se realizó la gestión del Fondo de Capital Semilla y el monitoreo de la ejecución de los recursos asignados. En el otorgamiento de los recursos del Fondo se utilizó el mecanismo de concursos. En las tres versiones del concurso que se realizaron -2013 a 2015- se otorgó un monto de hasta $\$ 450000000$ anuales, concediendo hasta $\$ 30000000$ por año a 15 proyectos de estudiantes y egresados de la Universidad EAN.

Con el fin de asignar los recursos se realizaron tres concursos en los años 2013, 2014 y 2015, los cuales se fueron mejorando en diversos aspectos, tanto en el proceso de inscripción como en las herramientas para la evaluación. En la segunda versión, para el primer filtro, se utilizó un formato de perfil de negocios - una versión simplificada del plan de negocios usado en la primera versión-; una vez superado este primer paso se desarrollaron talleres grupales sobre pensamiento de diseño y modelo de negocio, con una duración de cuatro horas cada uno, donde se dieron recomendaciones para profundizar el conocimiento del usuario y validar la propuesta de valor. Así mismo, herramientas como el lienzo de modelo de negocio -Canvas-y un simulalador financiero, para mejorar los perfiles de negocio y completarlos, con el propósito de esarrollar planes de negocio más estructurados y ser evaluados en el segundo filtro.

Adicionalmente, en la versión dos, se fortalecieron, mediante talleres grupales, a los emprendedores en habilidades comunicativas -Elevator Pitch- para la sustentación final de sus proyectos ante el Comité de Selección. Estos talleres se desarrollaron de forma práctica dentro de un proceso de identificación y apropiación de su modelo de negocios, junto con el

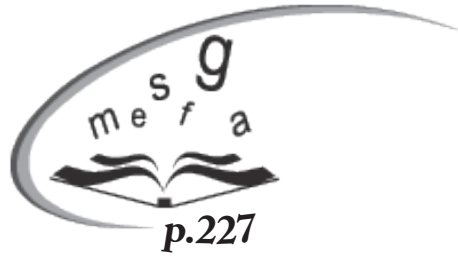
fortalecimiento de las capacidades comunicativas a partir de técnicas de creación de narrativas, respiración y gesticulación, que resultaron en discursos de negocios claros. El flujo del concurso en su segunda versión se muestra a continuación (Figura 2).

Figura 2. Flujo del concurso del Fondo de Capital Semilla Universidad EAN - versión 2.

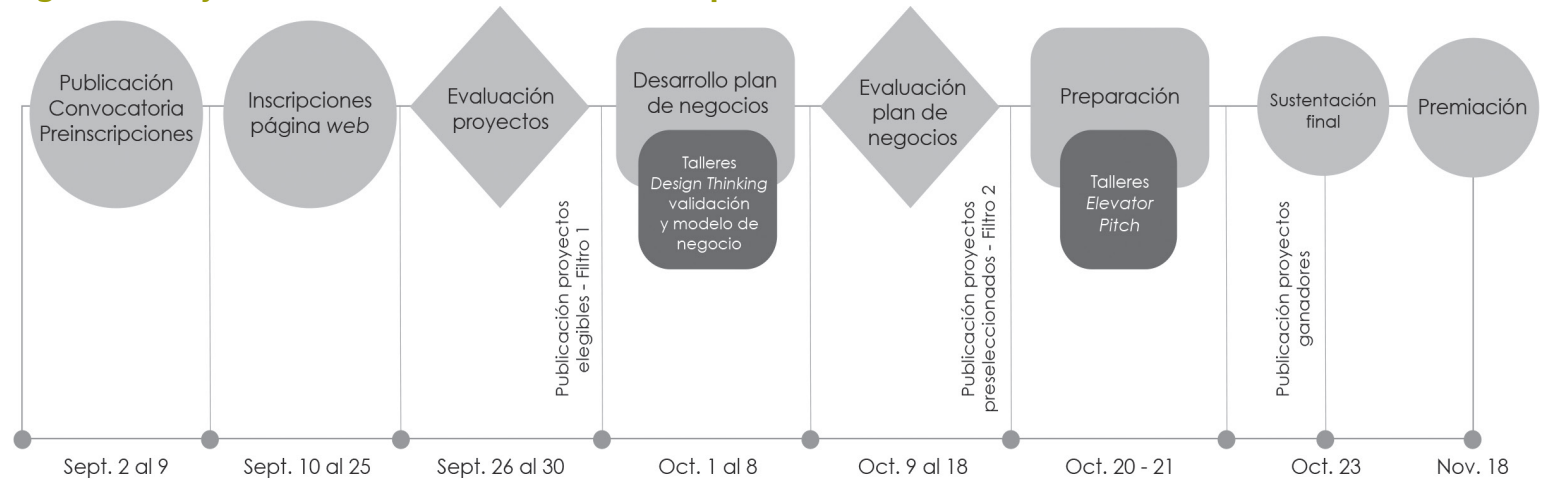

Fuente. Elaboración propia de los autores. 
La estrategia alrededor del acompañamiento a emprendedores no solo giró a partir de la asignación de recursos propios de la Universidad. En el 2014, el Instituto accedió a un proyecto de cofinanciación con iNNpulsaBancoldex para fortalecer las capacidades institucionales en la incubación y aceleración, partiendo de los aprendizajes del IN3, el PAE y el Fondo de Capital Semilla.

En el proceso se obtuvo transferencia de metodologías y buenas prácticas por parte de entidades con experiencia internacional en emprendimiento, como Incubatec Ufro -Chile-, Startup Essentials -LATAM- y Lever Laboratory S.A.S. Uno de los primeros ajustes fue el proceso de valoración de los perfiles de los emprendedores del Fondo de Capital Semilla, proceso que busca identificar habilidades blandas que aumenten las posibilidades de éxito en el desarrollo y puesta en marcha de sus iniciativas. Las habilidades observadas fueron el liderazgo, el compromiso, la escucha, el trabajo en equipo, la flexibilidad, la resiliencia, entre otros. Esta valoración de los perfiles de los emprendedores se realizó durante el desarrollo de un Bootcamp de tres días completos en emprendimiento ágil y validación temprana, que incluyó un filtro que evaluaba tanto el perfil de negocio presentado en la inscripción al concurso, como el perfil del emprendedor que se evidenciaba en su participación en el Bootcamp -filtro dos-. Adicionalmente, en la fase de entrenamientos, se profundizaron y extendieron los talleres, desarrollando uno de tres días sobre estrategia de innovación guiada por la marca -una metodología de descubrimiento de oportunidades de innovación basada en el pensamiento de diseño, la estrategia de marca y la validación temprana-; y un taller de ocho horas en modelo de negocio y finanzas para emprendedores, donde se ampliaron los contenidos desarrollados en la versión dos de duración y acompañamiento de más tutores - de dos tutores en la versión dos se pasó a tener seis tutores en la versión tres- durante el tiempo del taller para acompañar el mejoramiento de los modelos y planes de negocio. Finalmente, se incluyó una entrevista en el filtro tres, en la cual los emprendedores presentaron sus modelos de negocio reestructurados durante el Bootcamp y los entrenamientos en un pitch de cinco minutos seguido por diez minutos de preguntas y respuestas. El flujo de la tercera versión del concurso se puede observar más adelante (Figura 3).

Figura 3. Flujo del concurso Fondo de Capital Semilla Universidad EAN - versión 3.

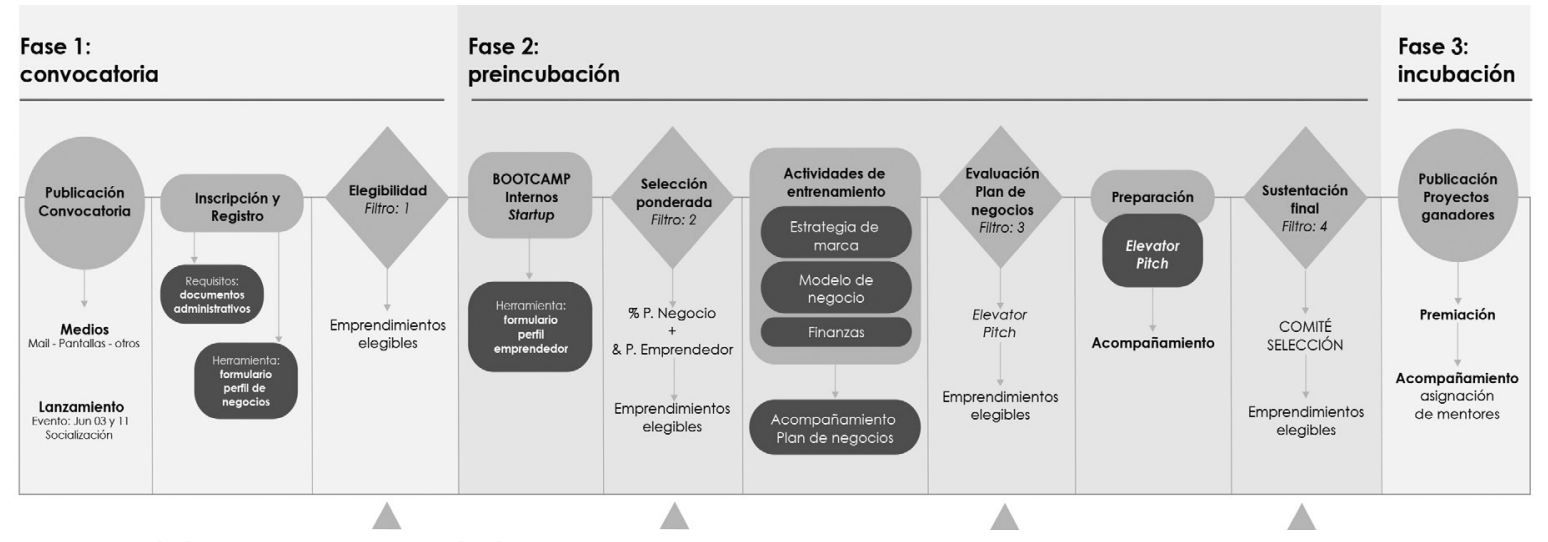

Fuente. Elaboración propia de los autores. 
Los recursos asignados y ejecutados en las tres versiones se pueden ver a continuación (Tabla 1). Durante las versión uno y dos del concurso se retiraron tres y un proyecto, respectivamente; argumentaron el retiro por la falta de tiempo para la ejecución del proyecto, lo cual resalta la importancia de evaluar el perfil del emprendedor y no solo del proyecto. Cabe anotar que en la versión 2015 solo se beneficiaron tres proyectos, dado un cambio en la política de asignación de recursos, que fue menor para el año 2015 en capital semilla entregado directamente a los emprendedores para disponer de más recursos para su acompañamiento y soporte. Este cambio se realizó debido a las siguientes razones: a) el rol de la Universidad dentrodelecosistemaemprendedor está orientado al fomento y acompañamiento de los emprendedores y a la ejecución de los recursos de capital semilla -su administración y seguimiento contable- desviaba el enfoque de la Universidad; b) existen fondos de capital semilla en el ecosistema con mayor impacto y con estructuras pertinentes y dedicadas a vigilar su ejecución de los mismos; c) el IDELI -actual Instituto para el Emprendimiento Sostenible-, logró gestionar fondos externos para los emprendedores, lo cual es un indicador importante que puede seguir mejorando si el acompañamiento se potencia.

Tabla 1. Recursos del Fondo de Capital Semilla ejecutados a junio de 2016.

\begin{tabular}{|c|c|c|c|}
\hline Semillero & $\begin{array}{l}\text { Valor } \\
\text { por girar }\end{array}$ & $\begin{array}{c}\text { Valor } \\
\text { desembolsado }\end{array}$ & $\begin{array}{l}\text { Valor por } \\
\text { desembolsar }\end{array}$ \\
\hline $\begin{array}{l}\text { Versión } 2013 \\
12 \text { beneficiarios }\end{array}$ & $\$ 360000000$ & $\$ 352463445$ & $\$ 7536555$ \\
\hline $\begin{array}{l}\text { Versión } 2014 \\
14 \text { beneficiarios }\end{array}$ & $\$ 420000000$ & $\$ 411569758$ & $\$ 8430242$ \\
\hline $\begin{array}{l}\text { Versión } 2015 \\
3 \text { beneficiarios }\end{array}$ & $\$ 90000000$ & $\$ 32263575$ & $\$ 57736425$ \\
\hline Total & $\$ 870000000$ & $\$ 796296778$ & $\$ 73703222$ \\
\hline
\end{tabular}

Fuente. Elaboración propia de los autores.

El proceso de asignación y ejecución de recursos se desarrolló bajo el acompañamiento para la supervisión del destino de los recursos, la validación y proyección del modelo de negocio y las acciones por seguir y así garantizar una puesta en marcha exitosa. Esta supervisión se realizó durante un año -en algunos casos el término se amplió debido a casos particulares donde el desembolso tomaba más tiempo- y a cada beneficiario se le asignó un tutor que no solo hacia seguimiento al desembolso de los recursos, sino también lo acompañaba en su proceso de fortalecimiento como emprendedor. Los emprendedores eran guiados en el proceso de consecución de recursos de otros fondos de capital, como Fondo Emprender del SENA, Emprender Paz, y de inversionistas ángeles y privados. Actualmente, se están terminando de ejecutar los recursos de los últimos beneficiarios.

La síntesis de los resultados del acompañamiento desde el 2011 a 2015, se presenta más adelante (Tabla 2). En los años 2011 y 2012 no se realizó un monitoreo al estado de los proyectos después de la fecha en la que se dio el acompañamiento, y del 2013 en adelante se tiene un seguimiento a los emprendedores beneficiarios del Fondo de Capital Semilla, quienes aún son activos y cercanos al Instituto para el Emprendimiento Sostenible. 
Tabla 2. Síntesis de resultados del acompañamiento realizado entre el 2011 y el 2015.

\begin{tabular}{|c|c|c|c|c|}
\hline Periodo & $\begin{array}{l}\text { Proyectos } \\
\text { inscritos }\end{array}$ & $\begin{array}{l}\text { Proyectos } \\
\text { asesorados }\end{array}$ & $\begin{array}{l}\text { Proyectos } \\
\text { con plan } \\
\text { de negocio } \\
\text { estructurado }\end{array}$ & Capital gestionado \\
\hline 2011 - IN3 & 45 & 26 & 11 & $\begin{array}{l}\text { Dos planes de negocios viabilizados para } \\
\text { financiación por el Fondo Emprender del } \\
\text { SENA. }\end{array}$ \\
\hline $2012-$ IN3 & 50 & 28 & 11 & $\begin{array}{l}\text { Nueve proyectos presentados a panel de } \\
\text { inversión - dos pasaron a segunda cita } \\
\text { con inversionistas privados. }\end{array}$ \\
\hline $\begin{array}{l}2013 \text { - PAE \& } \\
\text { Fondo de Capital } \\
\text { Semilla EAN }\end{array}$ & 100 & 60 & 18 & $\begin{array}{l}\text { Cuatro planes de negocios asignados para } \\
\text { financiación por el Fondo Emprender del } \\
\text { SENA -346M - ejecutados en el 2014-. } \\
12 \text { financiados por el Fondo de Capital } \\
\text { Semilla EAN -ejecutados en el 2014-- } \\
22 \text { empresas en funcionamiento. } \\
\text { Total: } 706 \text { millones gestionados para } \\
\text { emprendedores. }\end{array}$ \\
\hline $\begin{array}{l}2014 \text { - PAE \& } \\
\text { Fondo de Capital } \\
\text { Semilla EAN }\end{array}$ & 185 & 76 & 20 & $\begin{array}{l}\text { Tres proyectos beneficiarios del Fondo } \\
\text { de Capital Semilla Xue de la SDDE-24M-. } \\
\text { Un proyecto beneficiado por emprender } \\
\text { paz -60M-. } \\
\text { Dos planes de negocios asignados para } \\
\text { financiación por el Fondo Emprender } \\
\text { del SENA -129M - Ejecutados en el } 2015 \\
\text {-2016-. } \\
14 \text { financiados por el Fondo de Capital } \\
\text { Semilla EAN -ejecutados en el 2015- } \\
2016-\text {. } \\
\text { Total: } 633 \text { millones gestionados para } \\
\text { emprendedores. }\end{array}$ \\
\hline $\begin{array}{l}2015 \text { - PAE \& } \\
\text { Fondo de Capital } \\
\text { Semilla EAN }\end{array}$ & 239 & 129 & 50 & $\begin{array}{l}\text { Dos planes de negocios asignados para } \\
\text { financiación por el Fondo Emprender del } \\
\text { SENA-198M - por ejecutar en el 2016-. } \\
\text { Tres financiados por el Fondo de Capital } \\
\text { Semilla EAN -por ejecutar en el 2016-. } \\
\text { Total: } 288 \text { millones gestionados para } \\
\text { emprendedores. }\end{array}$ \\
\hline
\end{tabular}

Fuente. Elaboración propia de los autores.

Como se observa, cada año se han incrementado considerablemente los proyectos inscritos; esto se debe no solo al mejoramiento de los procesos de convocatoria realizados a los concursos del Fondo de Capital Semilla, también influye el voz a voz de los emprendedores que han sido beneficiados y el fomento que se le hace al emprendimiento en los módulos transversales de emprendimiento para todas las carreras impartidas en la Universidad EAN, en donde los docentes identifican proyectos y emprendedores, y los direccionan a los servicios de acompañamiento del Instituto. 
En el seguimiento realizado a los 29 emprendedores beneficiados por el fondo de capital en sus tres versiones -2013 al 2015-, en agosto de 2016, el $72 \%$ de las iniciativas se encuentran activas -consolidadas como empresa y en funcionamiento-, generando 58 empleos directos y 148 empleos indirectos, con ventas en el 2015 que ascienden a los $\$ 2249$ millones y ventas, en el primer semestre del 2016, por $\$ 1435$ millones. El $7 \%$ de las iniciativas se encuentran en etapa de prototipado y el $21 \%$ están inactivas. Los emprendedores que no fueron beneficiados con el fondo no se han monitoreado a la fecha, sin embargo, algunos de ellos han aplicado al nuevo modelo de acompañamiento -EAN Impacta- y se encuentran en diferentes fases del proceso.

Con base en la experiencia del IN3, el PAE y el Fondo de Capital Semilla de la Universidad EAN, el resultado final del proyecto de mejoramiento y fortalecimiento de capacidades para la incubación y aceleración cofinanciado por iNNpulsa-Bancoldex fue la conceptualización de un nuevo modelo de servicios de acompañamiento denominado EAN Impacta. Este se enfoca en la supervisión integral de iniciativas de emprendimiento para fortalecerlas y prepararlas, y aprovechar de manera más eficiente los fondos externos y los servicios prestados por las diferentes organizaciones y entidades del ecosistema emprendedor en Colombia y Latinoamérica. Desde 2016, con la inclusión de la sostenibilidad en la política general de la Universidad EAN, el IDELI se transformó en un organismo que integra la sostenibilidad, la innovación, el liderazgo y el emprendimiento, cambiando su nombre por el de Instituto para el Emprendimiento Sostenible, donde se incorporó el programa EAN Impacta, dentro de las acciones de extensión del Instituto.
El modelo construido incluye metodologías para todas las fases del proceso empresarial, para la solución de problemas complejos o para la generación de ideas y validación de modelos de negocios mediante el uso de técnicas de creatividad y procesos ágiles de desarrollo, las cuales se vienen incorporando en los programas de emprendimiento a nivel mundial. Además, se continúa utilizando la formulación de un plan de negocios como herramienta de gestión para la consecución de recursos y para la implementación. Para la etapa de aceleración se ha creado un proceso de consultoría donde participan equipos de docentes con experiencia en consultoría, estudiantes de maestría, como consultores junior y estudiantes de pregrado en procesos de práctica como asistentes de consultoría.

En todas las fases del proceso de desarrollo empresarial es indispensable identificar oportunidades, no solo en las fases iniciales, sino también procesos de validación de modelos de negocios, redefinición de la estrategia de la empresa madura y la ampliación de su portafolio de productos y servicios. En todas estas fases, la incorporación del pensamiento de diseño facilita procesos creativos y potencia resultados innovadores.

\section{El pensamiento de diseño es un enfoque de innovación sostenible basado en el uso de herramientas de diseño para integrar las necesidades de las personas (Deseable), las posibilidades de la tecno- logía (Factible) y los requerimientos para el éxito del negocio (Viable) (IDEO, 2016).}

El pensamiento de diseño está centrado en el usuario -Human Centered Design, HCD-, por lo cual las personas -usuarios y otros actores- están en el centro del proceso de principio a fin, para asegurar que las soluciones den respuesta a necesidades reales. 
El pensamiento de diseño está basado en un proceso de diseño secuencial de siete pasos (Ambrose y Harris, 2010), y cada uno plantea un momento y una acción por realizar, empezando por la definición del problema o situación y seguido por la investigación pertinente para determinar el contexto sobre el cual se desarrolla el problema definido.

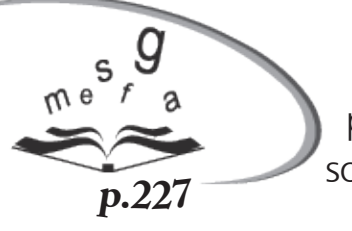

Se continúa con el momento más creativo del proceso, en el que se presentan soluciones alternativas, las cuales se prototipan para ponerlas a prueba y verificar si realmente se resuelve el problema, seleccionando una alternativa final para detallar. El penúltimo paso es la implementación y entrega de la solución que da respuesta al problema inicial, y finalmente, se tienen las lecciones aprendidas y la retroalimentación que ayuda a mejorar los procesos futuros y fomentar las iteraciones dentro del mismo proceso. Esto permite disminuir los riesgos gracias a la fase de prototipado, en la cual se ponen a prueba los productos y servicios antes de salir al mercado y ser comercializados.

El pensamiento de diseño se fundamenta en un proceso iterativo, en este sentido, hace conexión con el método Lean Startup, una adaptación de Eric Ries del método Lean Manufacturing o Lean Production. Consiste en una validación rápida de los procesos de los cuales se aprende, y de esta manera, acelerar la creación de un negocio validando cada paso, midiendo el progreso y mejorando con la retroalimentación de los clientes. Este método ayuda a los emprendedores a disminuir los riesgos, probando con prototipos y productos mínimos viables antes de hacer un gran lanzamiento al mercado. Es una de las herramientas más populares para desarrollar proyectos de innovación ligados al emprendimiento, especialmente, en ámbitos tecnológicos (Ries, 2011).
Cabe resaltar que estas metodologías se utilizan en las tres rutas del modelo propuesto en el Programa EAN Impacta, que se detallarán en los siguientes apartados. Adicionalmente, para la ruta de aceleración se usa una metodología de diagnóstico empresarial desarrollada en la Universidad EAN Ilamada Modelo de Modernización para la Gestión de las Organizaciones $\left(\mathrm{MMGO}^{\circledR}\right)$, con el objetivo de conocer, de forma integral, el estado de los aspectos principales de la empresa, como son finanzas, direccionamiento estratégico, exportaciones, gestión humana, transferencia del conocimiento e innovación. Con base en el diagnóstico se identifican las áreas de la empresa para fortalecer con la mentoría especializada y con la articulación con el ecosistema.

\subsubsection{Programa EAN Impacta}

La implementación del programa EAN Impacta se inició en marzo de 2016. A continuación se describe en detalle, con los pilares definidos desde su concepción, incluyendo algunos ajustes que se han desarrollado desde su puesta en marcha. Se espera que el modelo siga teniendo mejoramiento continuo a partir de las lecciones aprendidas en la implementación de cada fase y con las diferentes experiencias y casos que se van desarrollando.

El principal objetivo del programa es generar un proceso permanente de preincubación, incubación y aceleración de iniciativas de negocio, mediante servicios de acompañamiento en aspectos técnicos y de competencias blandas que garanticen la creación de empresas con gran potencial de crecimiento y emprendedores líderes comprometidos con la sostenibilidad social, económica y ambiental del país. 
Para cumplir con este objetivo, se ofrece a los emprendedores servicios de acompañamiento para la incubación de modelos de negocio con énfasis en los aspectos de la sostenibilidad en sus tres aristas: económica, social y ambiental, y con estrategias para potenciar su crecimiento en ventas y generación de empleos. En este proceso de acompañamiento se identifican aquellos emprendimientos con modelos de negocios sostenibles y con gran potencial de crecimiento para apoyarlos en la gestión de recursos financieros en el proceso de su puesta en marcha y consolidación. Paralelamente, se identifican y articulan los diferentes actores del ecosistema de emprendimiento del país con los emprendedores EANistas y así aprovechar los servicios y beneficios que les puede ofrecer este ecosistema (Figura 4).

Figura 4. Esquema general programa EAN Impacta.

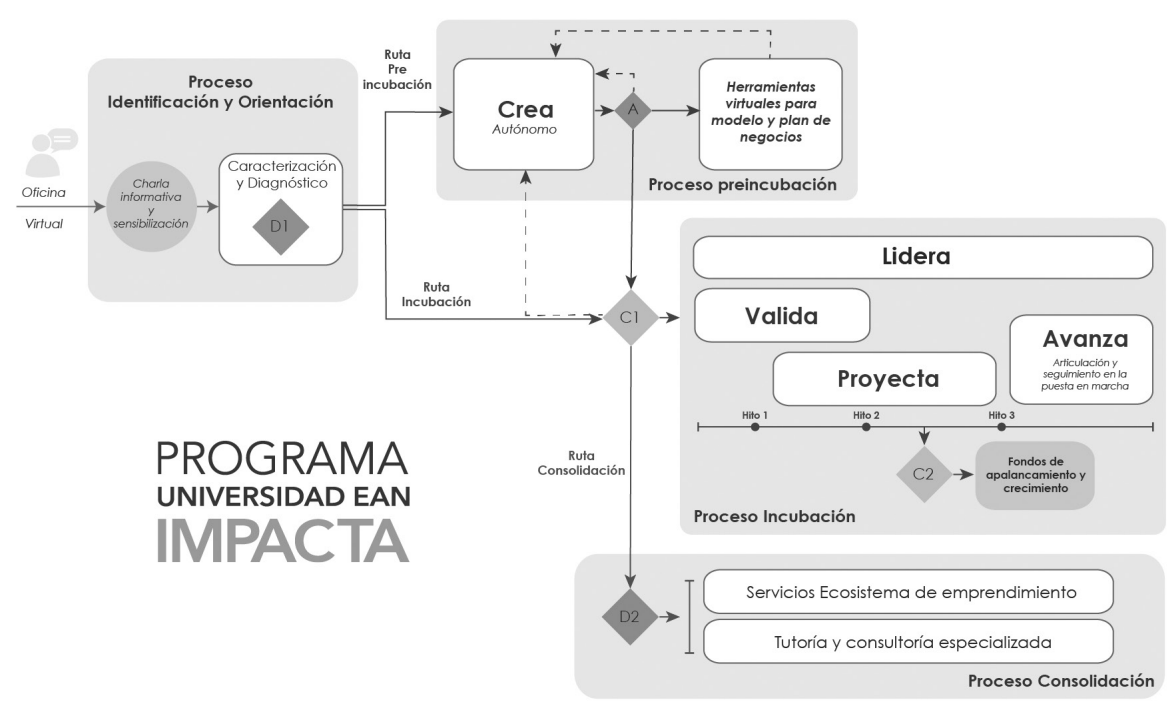

Fuente. Elaboración propia de los autores.

A partir de un proceso de identificación y diagnóstico -D1-, los emprendedores que aplican al programa son orientados a las rutas de preincubación, incubación y aceleración, según el grado de desarrollo de sus iniciativas y la evidencia de componentes de sostenibilidad e innovación en sus propuestas.

La ruta de preincubación se constituye principalmente por el programa CREA, que a través de metodologías de pensamiento de diseño, emprendimiento ágil y validación temprana, los emprendedores redefinen su idea de negocio teniendo en cuenta la identificación de problemáticas y oportunidades del mercado basadas en un análisis profundo del contexto y de los usuarios potenciales, la posterior generación de alternativas de solución y la realización de experimentos que permitan al emprendedor validar sus ideas y definir una propuesta de valor de la cual se pueda partir para el desarrollo de un modelo de negocio. Al finalizar el programa CREA, los tutores que han realizado seguimiento a los proyectos dan un concepto y avalan -A- aquellos proyectos que se postulan al comité de incubación -C1- para continuar en la siguiente fase del programa. 
Los proyectos que son postulados y enrutados al proceso de incubación -C1-, tanto en el aval de CREA -A- como en el proceso de diagnóstico -D1-, son evaluados por el comité de incubación. Si son avalados por este, se direccionan al proceso de incubación de empresas, el cual se basa en el acompañamiento por parte de tutores coach y especializados, para validar sus modelos de negocio -valida- y proyectarlos en las áreas de mercadeo, organizacional, operativa y financiera -proyecta-, con el fin de crear y consolidar empresas sostenibles con gran potencial de crecimiento.

La ruta de aceleración busca articular a las empresas que se encuentren en una etapa de madurez, con mentores y tutores especializados y con los actores del ecosistema pertinentes, de acuerdo con las necesidades de cada empresa detectadas a partir de un diagnóstico empresarial -D2-.

Durante todo el proceso de los emprendimientos en las diferentes fases del programa EAN Impacta se les hace seguimiento, llevando la trazabilidad del estado de cada uno, el cumplimiento de hitos -los hitos son definidos por el emprendedor y sus tutores, según cada caso- y sus indicadores de crecimiento, como la generación de empleos, las ventas y gestión de recursos financieros, entre otros. Esto se realiza a través del monitoreo de una base de datos maestra administrada por la coordinación del programa y de los soportes de cumplimiento de fases e hitos que se recolectan en las diferentes plataformas de acompañamiento y seguimiento utilizadas en cada fase y que reposan de manera digital en carpetas organizadas por proyecto y por etapa. Los indicadores que se miden en el proceso son: emprendedores que inician y terminan cada fase del proceso de acompañamiento, empresas creadas, empresas activas-en el monitoreo semestral que se sigue realizando una vez terminan el proceso de acompañamiento-, recursos de capital o financiación gestionados, etc. Los indicadores que se miden de los emprendedores acompañados son: empleos generados -directos e indirectos-, ventas, recursos de capital o financiación gestionados, participación en eventos comerciales, entre otros.

\section{- Parte 1. Proceso de identificación y orientación}

Figura 5. Esquema del proceso de identificación y orientación.

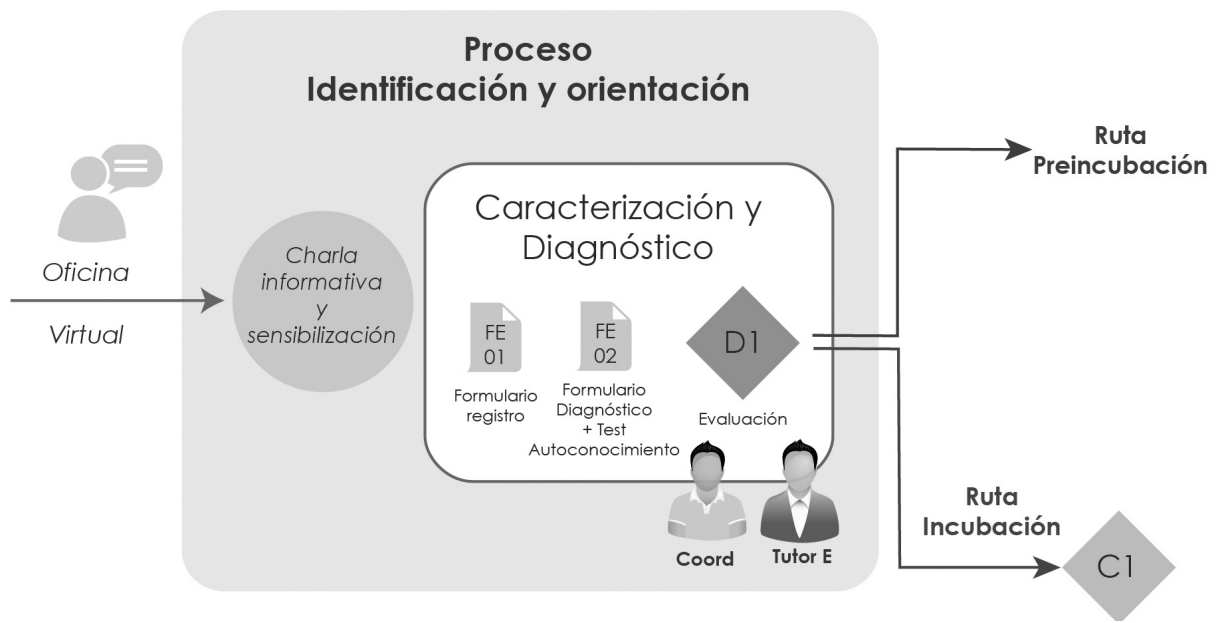

Fuente. Elaboración propia de los autores. 
Es el proceso mediante el cual se diagnostican y direccionan las iniciativas empresariales que van a ser acompañadas en el programa. Es importante destacar que este proceso incluye proyectos que se encuentran en etapa de idea de negocio, modelo de negocio, plan de negocio y empresas en marcha.

El proceso de identificación y orientación inicia con la inscripción del emprendedor EANista en la base de datos maestra del Instituto para el Emprendimiento Sostenible, que se puede realizar de manera personal o por correo electrónico, para ser invitado a la charla informativa que se realiza mensualmente o se puede ver a través de un enlace web. Posteriormente, el emprendedor debe diligenciar los formularios de registro y caracterización, los cuales permiten diagnosticar si se trata de una idea, un modelo, un plan de negocios o una empresa en marcha. La recepción de estos formatos se hace de manera bimensual. La caracterización permitirá que el coordinador y mentor identifiquen el estado actual del proyecto presentado y emitan un concepto técnico al respecto; este diagnóstico se realiza en el término de dos semanas una vez recibidos los formularios -Diagnóstico 1-D1-. El proceso termina orientando cada caso al siguiente proceso que puede ser: ruta de preincubación, comité de incubación o ruta de aceleración. En total, el proceso de identificación y orientación puede durar uno a dos meses, según el momento en el que el emprendedor realice la inscripción en la base de datos.

\section{- Parte 2. Ruta preincubación}

Figura 6. Esquema de la ruta Preincubación.

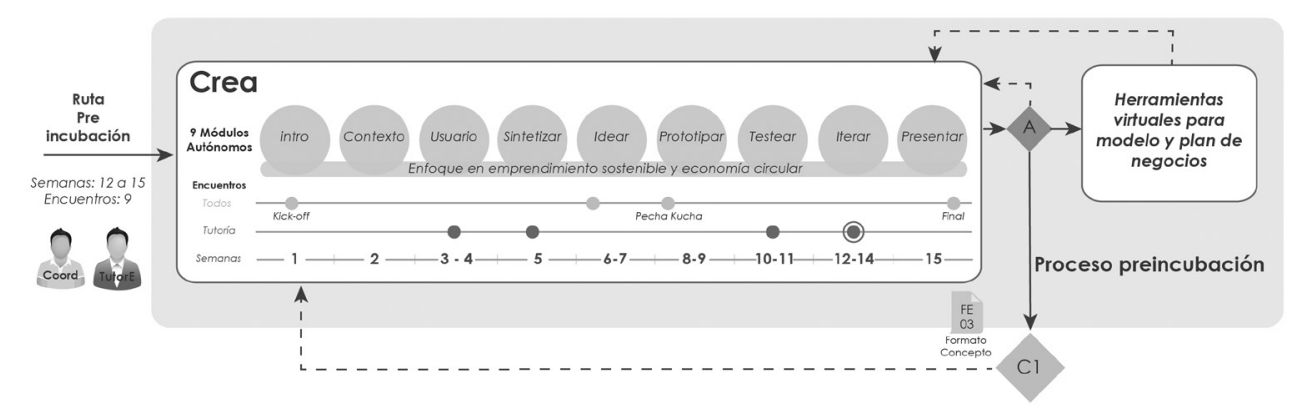

Fuente. Elaboración propia de los autores.

La preincubación busca estructurar y definir una idea de negocio, a través de la identificación de problemáticas y oportunidades del mercado, el desarrollo de ideas que respondan a ellas y la realización de experimentos que permitan al emprendedor validar sus ideas y definir una propuesta de valor de la cual se pueda partir para el desarrollo de un modelo de negocio.
La fase CREA está basada en metodologías de pensamiento de diseño e innovación, impulsada por la marca -Brand Driven Innovation-, y metodologías de emprendimiento ágil y validación temprana. Esta inicia con la exploración y enfoque de oportunidades y problemáticas; continúa con el proceso de ideación de soluciones, posteriormente con la selección de ideas para ser prototipadas y validadas en un proceso iterativo, hasta la 
estructuración de una idea final y la definición de su propuesta de valor.

La fase CREA se desarrolla a través de un programa de formación y acompañamiento mixto -blended-en donde los emprendedores desarrollan nueve módulos virtuales de manera autónoma, con espacios de socialización con tutores especializados para presentar los avances. Estos espacios se realizan como tutorías virtuales de dos horas, por grupos de tres a cuatro emprendedores, y se realizan en los módulos tres -Análisis de usuario-, cuatro -Síntesis de hallazgos-, siete -Validación- y ocho -Iteración-. En algunos casos se dan dos sesiones de tutoría en el módulo ocho cuando los proyectos realizan varias iteraciones. Igualmente, se establecen espacios de socialización del proceso entre emprendedores para que exista colaboración entre aquellos que están viviendo el proceso. Los espacios presenciales grupales se realizan en los módu-los uno Introducción-, cinco -Ideación-, seis -Prototipado- y nueve -Presentación-.
Una vez los emprendedores tengan los entregables de todos los módulos, los exponen en una sesión final de presentación -en el último módulo una vez han desarrollado todos los anteriores e iterado las veces necesarias-, donde los mentores especializados realizan la evaluación y emiten el concepto de aval. Si el mentor da visto bueno al proceso desarrollado -aval - (A)-, el emprendedor es presentado al comité de incubación -C1-, que se realiza cada mes, donde expone su modelo de negocio reestructurado. Si no se da visto bueno, el emprendedor puede repetir el proceso de preincubación de manera autónoma y exponer de nuevo sus resultados en la presentación final del siguiente grupo de proyectos o continuar con herramientas virtuales para el desarrollo del modelo y el plan de negocios -sin acompañamiento de tutores-, las cuales son facilitadas vía email, donde encuentran instructivos para el desarrollo de sus modelos de negocio. La fase CREA se desarrolla de 12 a 15 semanas, según la velocidad de los proyectos en curso.

\section{- Parte 3. Ruta incubación}

Figura 7. Esquema de la uta de Incubación.

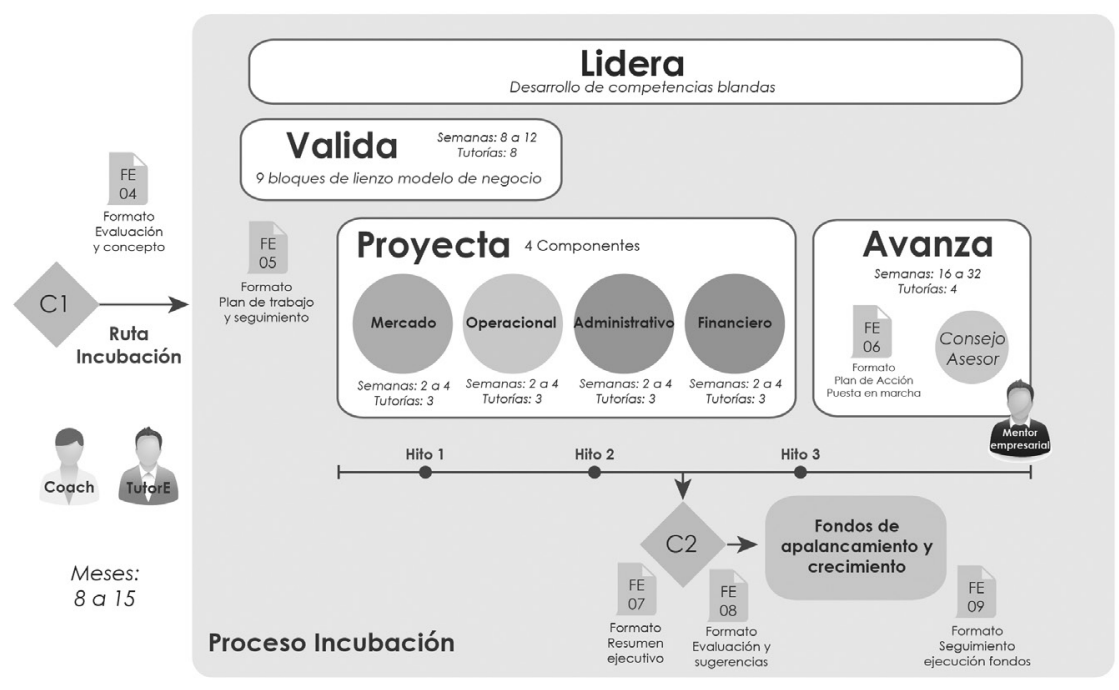

Fuente. Elaboración propia de los autores. 
La incubación de empresas es el proceso de acompañamiento a emprendedores EANistas, mediante el cual se formulan y validan modelos y planes de negocio con el fin de crear y consolidar empresas sostenibles con potencial de alto impacto.

El proceso de incubación inicia con una propuesta de valor validada, la cual permite la definición y aprobación del modelo de negocio en la fase valida. Continúa con la estructuración del modelo de negocio en los temas: comercial, técnico y operativo, organizacional, financiero y de inversión, en la fase proyecta; y finaliza con la implementación del plan operativo y puesta en marcha del emprendimiento, en la fase avanza. Adicionalmente, el emprendedor recibirá herramientas para la reflexión y desarrollo de habilidades blandas, en la fase lidera.

El proceso de incubación inicia con el aval del comité de incubación -C1-, que asigna el tutor líder y diseña el plan de trabajo con el emprendedor; y determina las necesidades de tutoría en temas especializados, según el caso de cada proyecto. Durante las fases valida, proyecta y lidera, el tutor realiza el seguimiento para lograr la ejecución del plan de trabajo definido y el cumplimiento de hitos por parte del emprendedor. Así mismo, se espera que durante el proceso de validación y proyección se generen las primeras ventas. Los hitos son los logros críticos del emprendedor que identifican momentos clave y que llevan a la empresa al siguiente nivel. Estos se refieren a los indicadores de tracción que se definen para la iniciativa, como terminación de un prototipo, un número de clientes definido, contratos nuevos, etc.

\subsubsection{Fase I. Valida}

En esta fase del proceso de incubación, el emprendedor formula y valida el modelo de negocio utilizando la metodologías Lean Startup. Se elabora de manera blended, a través del desarrollo autónomo de ocho módulos, que inician con la definición del modelo de negocio y que van guiando al emprendedor en el proceso de validación de cada uno de sus componentes; así mismo, facilita herramientas necesarias para realizar una validación completa y exitosa. Durante este proceso se realizan tres sesiones presenciales grupales -pueden ser cuatro a ocho proyectos- de dos horas, en los módulos uno -valora-, cinco -comunica- y ocho -cierra-. Adicionalmente, se realizan tutorías individuales de dos horas a cada proyecto en los módulos dos -entrega-, tres -captura-, cuatro -prototipa-, seis -Ejecuta- y siete -proyecta-. A estas tutorías pueden asistir los emprendedores de manera virtual o presencial.

En cada uno de los módulos se trabajan iteraciones para ir validando los nueve bloques del lienzo del modelo de negocios de Alexander Osterwalder; además, los participantes se suscriben a un curso de libre acceso en Internet: How to build a startup, de Steve Blank. En el módulo valora, se plasma el primer modelo de negocio concebido en el Canvas, de propuesta de valor y del modelo de negocio. En los módulos entrega y captura se realizan iteraciones para validar todo el lado derecho del Canvas del modelo de negocio -segmento, propuesta de valor, canales, relacionamiento y modelo de ingresos-. En el módulo prototipa se realizan iteraciones basadas en prototipos más estructurados que representen el lado derecho del Canvas del modelo de negocio. En el módulo comunica se hace una presentación de avance ante tutores -jurados- para dar aval de esta mitad del Canvas. En los módulos ejecuta y proyecta, se realizan iteraciones para validar 
el lado izquierdo del Canvas del modelo de negocio -recursos, aliados, actividades claves, modelo de costos, y gastos y alternativas de financiación-. Finalmente, se presenta, ante el comité de incubación, el modelo de negocio al que se llega en el proceso de validación, para dar aval y continuar al proyecta (Figura 8).

Figura 8. Esquema de la fase valida.

\section{Valida pran de Trabajo}

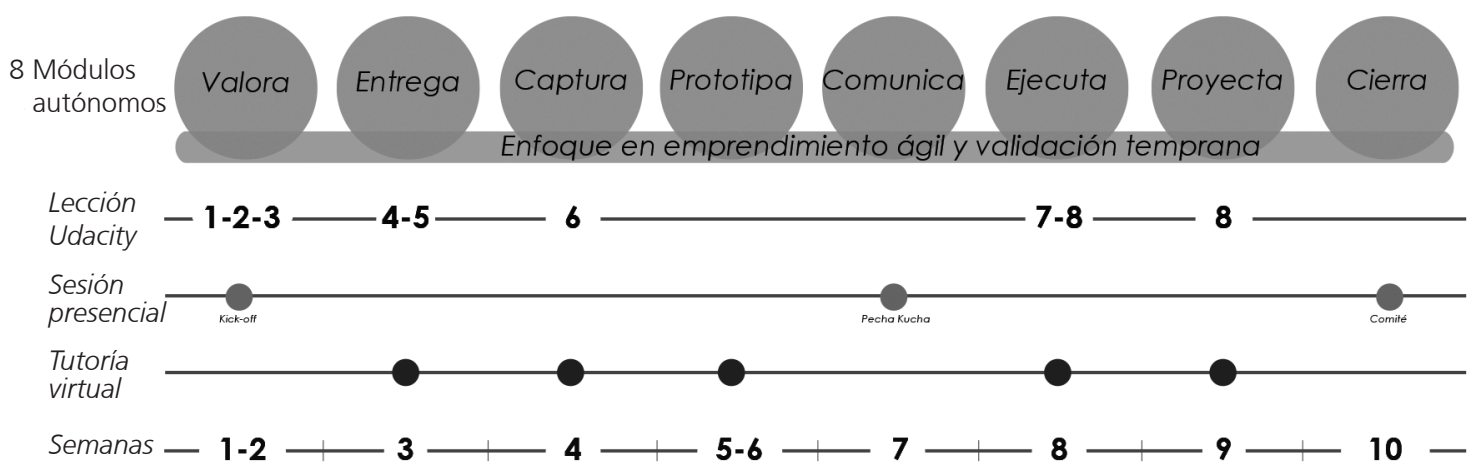

Fuente. Elaboración propia de los autores.

El producto final de este proceso debe ser el modelo de negocio validado, que demuestre articulación y coherencia entre la propuesta de valor y el mercado. Este proceso de validación se debe documentar, ya que es el insumo para la proyección del modelo de negocio y de la empresa, para lo cual el desarrollo de los módulos incluye un seguimiento documental de los formatos facilitados. El proceso valida se desarrolla entre 10 a 12 semanas, según la velocidad de los emprendedores.

\subsubsection{Fase II. Proyecta}

En esta fase se pretende asesorar al emprendedor en la proyección de su modelo de negocio, para detallar el funcionamiento de la empresa, prepararse para la puesta en marcha y gestionar la consecución de recursos dentro del ecosistema de emprendimiento.

Para la proyección del modelo de negocio se utilizará como insumo el aprobado en la fase valida, y la documentación utilizada en dicho proceso. En proyecta, el emprendedor trabaja uno a uno con un tutor coach asignado por el comité de incubación -C1-, durante cuatro meses, en los que se encuentran presencialmente o de manera virtual, si es necesario, en 12 sesiones de tutoría de máximo dos horas cada una.

Los aspectos que deben tenerse en cuenta para la proyección del modelo de negocio, son los componentes de mercado, producción, administrativos, y, finalmente, un componente financiero que evalúe la rentabilidad de la empresa. Con el desarrollo de estos aspectos, el emprendedor tendrá la información para escribir su plan de negocios, y así, alcanzar la consecución de fondos de inversión cuando sea requerido.

En la fase proyecta, el emprendedor desarrolla un plan de negocios detallado y coherente que es evaluado, ya sea por otros tutores del Instituto o sometido a una revisión profunda, cuando es presentado a posibles financiadores, 
como el Fondo Emprender del SENA. En esta parte, el programa EAN Impacta acompaña directamente a los emprendedores en la preparación de sus planes de negocio para aplicar a estos fondos, y en el caso del Fondo Emprender, cuando son asignados recursos, el programa EAN Impacta hace seguimiento a la ejecución de recursos, asignando un gestor técnico y operativo, según los requerimientos de dicho Fondo.

\subsubsection{Fase III. Lidera}

Esta es una fase transversal al proceso de incubación y puede desarrollarse en paralelo con las fases valida, proyecta y avanza. En esta fase, los emprendedores desarrollan de manera autónoma nueve módulos temáticos, en los que encontrarán material teórico, de inspiración e instrucciones para reflexión personal.

Durante el desarrollo de los módulos, los emprendedores tendrán unas sesiones de encuentro con un tutor especializado en liderazgo, con quien revisarán sus reflexiones personales para identificar elementos por mejorar y definir estrategias para desarrollar aquellas habilidades débiles. Estas sesiones son personalizadas, dos por emprendedor, durante el tiempo en el que están realizando las fases valida y proyecta. Adicionalmente, se invita a los emprendedores a participar en talleres grupales que se definen según las necesidades de los emprendedores en proceso de acompañamiento.

Algunos temas que se abordan en los módulos y talleres son: autoconciencia, propósito, comunicación, liderazgo humilde, transparencia, resiliencia e intuición, perseverancia, control, negociación, entre otros.

\subsubsection{Fase IV. Avanza}

Es la última fase del proceso de incubación, en la que se monitorea el resultado del proceso de la puesta en marcha de la empresa que se ha venido gestando desde el proceso de validación.

Esta fase tendrá en monitoreo al emprendedor. Esta actividad se realizará con un esquema de gobernanza, asignándole un comité asesor a cada una de las empresas. Aquí, el tutor y el emprendedor diseñan un plan para la puesta en marcha del proyecto, basado en los componentes desarrollados en la fase proyecta. Se realizan cuatro sesiones del comité asesor, de una hora y media cada una, con un espacio de dos meses entre ellas. Este lo confoman por el tutor coach, el director del Instituto para el Emprendimiento Sostenible y un tutor empresarial designado -parte de la red de mentores que se encuentra desarrollando el Instituto-.

Durante cualquiera de las fases del proceso de incubación, el tutor puede postular el proyecto ante el Comité de Fondos de Apalancamiento y Crecimiento -C2 (Figura 4 y Figura 7), que evaluará el proyecto mediante el análisis de dos documentos presentados por el emprendedor: resumen ejecutivo y plan de inversión. El comité de fomento está compuesto por el director del Instituto para el Emprendimiento Sostenible, un tutor diferente al tutor coach del emprendimiento -de cualquier facultad-, dos directivos de la Universidad EAN -vicerrectores, miembros de la Sala General o miembros del Consejo Superior- y otros invitados que se propongan. Este se reunirá cuando haya emprendedores postulados. 
Los proyectos que se presenten al Comité de Fondos de Apalancamiento deberán tener validados los nueve bloques del modelo de negocio, haber desarrollado por lo menos un componente de la proyección del modelo de negocios que justifique una necesidad puntual y que sea evidenciado con cumplimiento de hitos pactados en su plan de trabajo desarrollado con el tutor coach. Además, debe evidenciar el desarrollo de habilidades blandas, lo cual será avalado por el tutor coach y el tutor especializado de lidera.

El comité debe emitir un concepto acerca del nivel de los emprendimientos y de las oportunidades de mejora identificadas, hacer recomendaciones al emprendedor y al emprendimiento, verificar si cumple con los criterios para ser beneficiario de recursos de fomento destinados al apalancamiento y gestión de iniciativas empresariales de la Universidad EAN -evolución del Fondo de Capital Semilla anterior, el cual cuenta con recursos de capital de apalancamiento que se definen cada año, siendo, en 2016 , de $\$ 150$ millones- e identificar e informar sobre otros fondos a los que se puede presentar el emprendimiento; también, avalar su presentación en el caso de convocatorias abiertas en el momento en que se desarrolle el Comité.
Los recursos de fomento destinados al apalancamiento y gestión de iniciativas empresariales EANistas brinda recursos no condonables para apoyar dichas iniciativas en la preparación de su puesta en marcha y crecimiento, por lo cual serán destinados para inversiones asociadas al desarrollo de sus productos, servicios y mercado, como:

- Participación en talleres de entrenamiento sobre la creación y validación de modelos de negocio ante clientes.

- Consultoría especializada para la estructuración y toma de decisiones empresariales de carácter financiero y legal.

- Asesoría técnica para el diseño de productos, servicios o sistemas que hagan parte esencial de su modelo de negocio.

- Asesoría especializada en temas como planeación estratégica, coaching comercial, estrategias financieras, marketing y habilidades gerenciales, para la puesta en marcha y articulación con el ecosistema.

- Acompañamiento en el proceso para mejorar la presentación del informe financiero y el discurso ante los posibles inversionistas.

- Asesoría integral para el fortalecimiento del proyecto empresarial con énfasis en el mejoramiento del producto/servicio y crecimiento en ventas. 


\section{- Parte 4. Ruta aceleración}

Figura 9. Esquema de la ruta Aceleración.

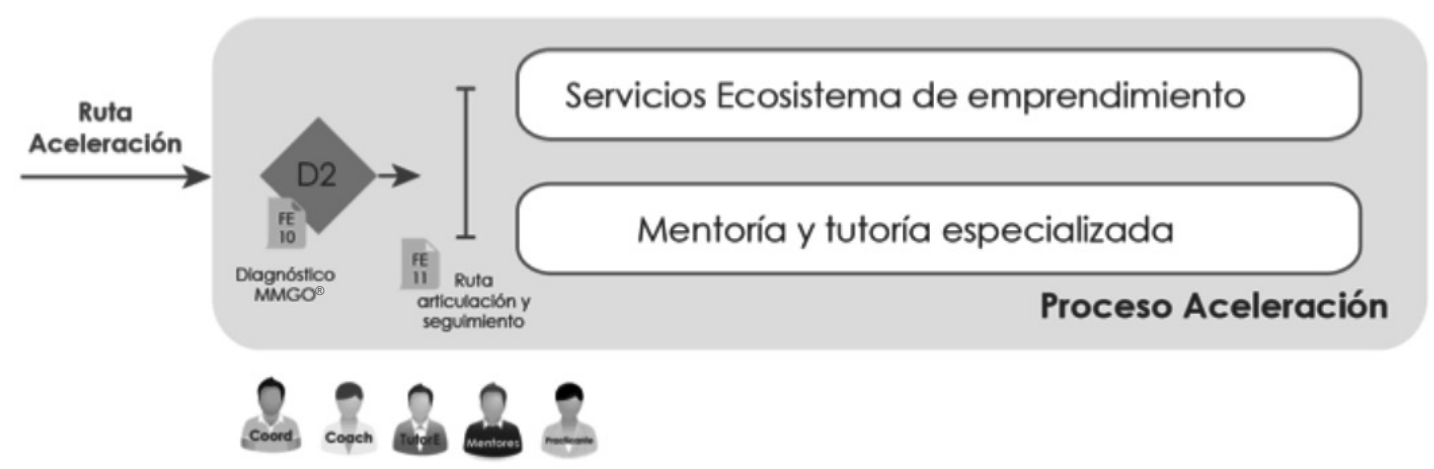

Fuente. Elaboración propia de los autores.

Teniendo en cuenta el ciclo de desarrollo empresarial en el marco de la política pública colombiana, el rol de la Universidad y la participación de sus diferentes actores de emprendimiento, la Universidad ofrece a sus estudiantes y egresados el servicio de articulación con los actores del ecosistema, de acuerdo con las necesidades de cada empresa.

En esta etapa, la empresa maximiza su valor agregado a través de la internacionalización y la innovación, y orienta sus esfuerzos hacia la consolidación de su crecimiento sostenido, mediante la búsqueda de socios estratégicos y nuevos clientes a través de redes de contactos internacionales y atracción de capital.

El proceso de aceleración de empresas del programa EAN Impacta inicia con el desarrollo de un diagnóstico empresarial detallado -D2-, en el cual se trabajará un plan de articulación con el ecosistema de emprendimiento -servicios y ofertas de otras entidades en el ecosistema-, y dado el caso, mentoría y tutoría especializada, brindada de forma presencial o virtual por tutores expertos que fungen como consultores líderes, estudiantes de maestría en gestión y dirección de empresas e innovación, quienes serán consultores senior, y estudiantes en práctica académica, que acompañarán el proceso.

El diagnóstico empresarial -D2- se realiza utilizando el Modelo de Modernización para la Gestión de Organizaciones $\left(\mathrm{MMGO}^{\circledR}{ }^{\circledR}\right.$, cuyo objetivo es la identificación de las actividades que conformarán la ruta de cambio, mejoramiento y reconversión hacia las mejores prácticas en la organización. Con la aplicación del modelo se define una ruta estratégica de articulación con los diferentes actores del ecosistema de emprendimiento y las necesidades de mentoría especializada para cada caso.

El emprendedor, en compañía de su tutor, debe generar un plan de seguimiento a seis meses para que se evalúen los impactos que las acciones han tenido sobre el desarrollo de la empresa. 


\subsubsection{Resultados EAN Impacta}

El programa EAN Impacta inició operaciones en febrero de 2016, recibiendo más de 30 proyectos mensuales de emprendedores interesados en el acompañamiento y la gestión de fondos. En seis meses de operación se han realizado cuatro charlas informativas y de sensibilización, donde se ha socializado a los emprendedores, el nuevo modelo, con el fin de alinear expectativas y comenzar el proceso de aplicación y diagnóstico; de esta forma, direccionar a los emprendedores a las diferentes rutas. Adicionalmente, se ha gestionado la participación de los emprendedores en diferentes eventos y convocatorias, logrando la adjudicación de capital semilla en la primera convocatoria del Fondo Emprender, del 2016. A continuación, se detallan los logros obtenidos hasta agosto de dicho año (Tabla 3).

Tabla 3. Resultados EAN Impacta de febrero a agosto de 2016.

\begin{tabular}{|c|c|}
\hline Tipo & Logros \\
\hline Acompañamiento & $\begin{array}{l}240 \text { emprendedores interesados en acompañamiento. } \\
88 \text { proyectos finalizan el proceso de inscripción. } \\
30 \text { emprendedores enrutados a preincubación. } \\
20 \text { emprendedores enrutados a incubación. } \\
1 \text { emprendedor enrutado a aceleración. } \\
37 \text { en ruta de aceleración. } \\
3 \text { tutores en preincubación. } \\
7 \text { tutores coach en incubación. } \\
31 \text { tutores especializados de otras facultades apoyan el programa. }\end{array}$ \\
\hline $\begin{array}{l}\text { Gestión de recursos para } \\
\text { los emprendedores }\end{array}$ & $\begin{array}{l}\text { Ocho planes de negocio presentados al Fondo Emprender. } \\
\text { Un plan de negocio beneficiado con } 75 \text { millones por el Fondo Emprender. } \\
\text { Dos proyectos con fondos gestionados por el } 60 \% \text { del proceso de } \\
\text { patente - } \$ 6375000) \text {, aportados por CONNECT BOGOTÁ para cada } \\
\text { proyecto - un proyecto ya en ejecución, un proyecto por firmar contrato-. }\end{array}$ \\
\hline $\begin{array}{l}\text { Participación en eventos y } \\
\text { convocatorias }\end{array}$ & $\begin{array}{l}\text { Cinco emprendedores asisten a la Feria de Jóvenes Emprendedores de la } \\
\text { Cámara de Comercio de Bogotá en abril de } 2016 . \\
30 \text { estudiantes y egresados asisten al Foro Líderes y Emprendedores en } \\
\text { la U. } \\
10 \text { proyectos inscritos en el Premio Estudiante Emprendedor. Un proyecto } \\
\text { queda entre los seis finalistas. } \\
\text { Un proyecto llega a semifinal del Hult Prize en USA. } \\
\text { Tres proyectos en Concurso de Empresas Sociales del JCI. } \\
\text { Un proyecto aplica al premio Everis Semana. } \\
\text { Un proyecto en Misión Impacto } 2 \text { de RCN. } \\
\text { Tres proyectos aplican a Apps.co. } \\
\text { Ocho proyectos aplican al concurso Ventures. }\end{array}$ \\
\hline
\end{tabular}

Fuente. Elaboración propia de los autores. 


\section{Conclusiones}

$\Delta$

manera de conclusión, se muestra que el apoyo a emprendedores EANistas a lo largo de los últimos seis años, ha venido evolucionando. Por un lado, debido al aumento de la demanda de atención por parte de los emprendedores, originado en el proceso de formación transversal en emprendimiento; y de otro lado, por la dinámica de la generación de conocimientos, tendencias y nuevas metodologías que se están utilizando para atender a los emprendedores, dados los cambios en los nuevos entornos económicos a nivel mundial (Figura 10).

Figura 10. Línea de tiempo de programas de incubación en la Universidad EAN.

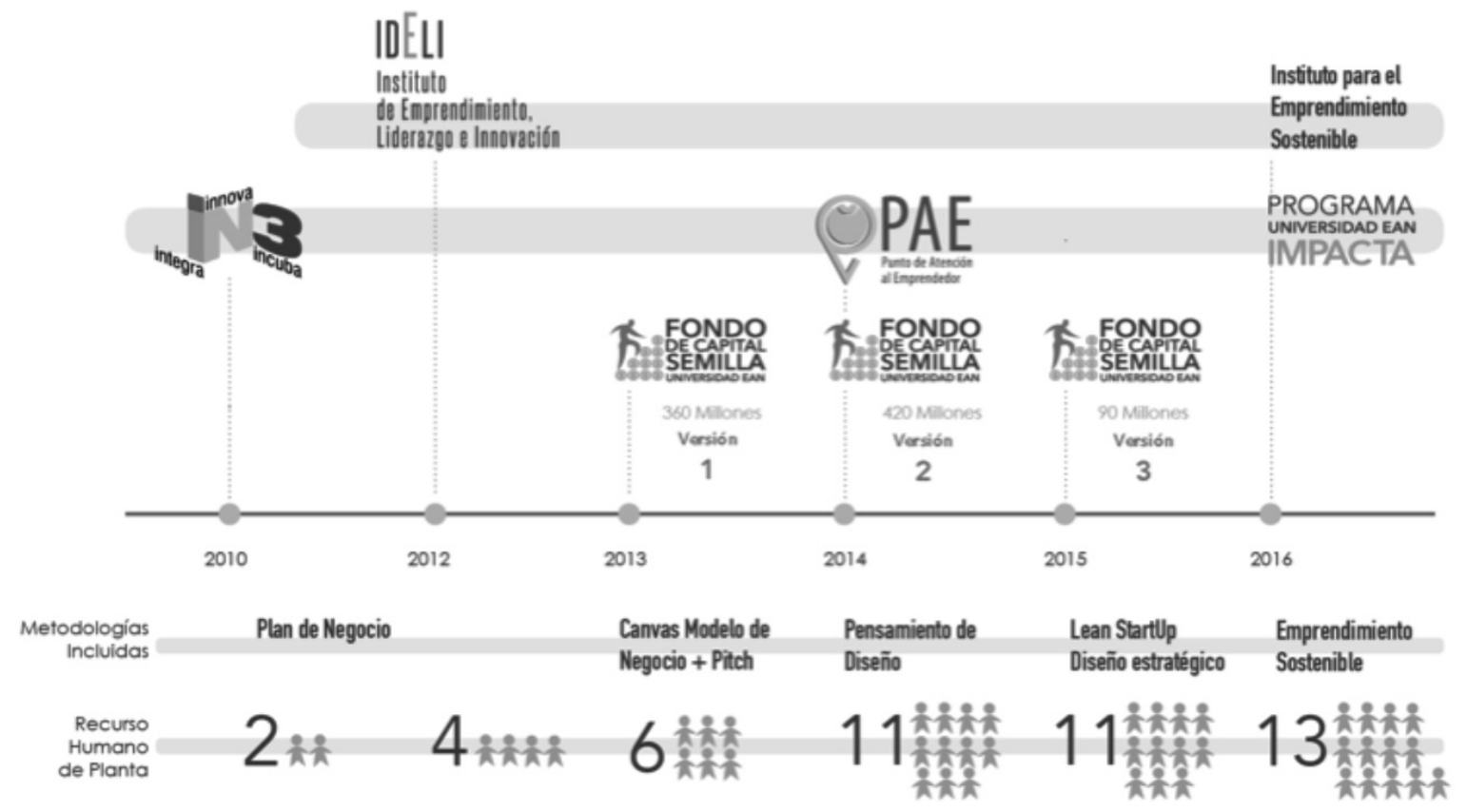

Fuente. Elaboración propia de los autores.

El reto de la incubación de empresas es una labor compleja, pues para que sea eficiente y pertinente se deben involucrar a todos los actores, los emprendedores, los docentes, los asesores, las empresas, las redes y las instituciones, dentro del ecosistema que favorezca al emprendedor. Por tal razón, el éxito radica, en buena parte, en lograr un trabajo coordinado y orientado a un fin común con las instituciones externas a la Universidad, enfocando, sobre todo, al financiamiento, que se convierte en un reto más complejo, dada las falencias del ecosistema colombiano sobre la consecución de recursos para los emprendedores.

Las experiencias aprendidas a lo largo de los últimos diez años en el proceso de incubación empresarial pueden desglosarse en las diferentes etapas del proceso. Como se observó (Figura 10), en 2010 se estructuró el programa de IN3, con resultados positivos comparados con lo anterior, pero distantes de lo que realiza 
una incubadora de talla mundial. Sin embargo, en esta primera etapa surgieron varios aprendizajes que le aportaron al programa EAN Impacta: el primero fue la necesidad de contar con una infraestructura más adecuada para un óptimo acompañamiento empresarial, aunado a procesos de ensayo y error por parte de los emprendedores antes de implementar su negocio. El segundo aprendizaje fue sobre el tipo de negocios que tenían una mayor probabilidad de perdurar en el tiempo, los cuales estaban asociados con sectores difusores de conocimiento, donde este se convirtió en un valor estratégico e integrado por equipos interdisciplinarios. Dicho aprendizaje se corroboró con investigaciones que involucraron empresas establecidas, las cuales se hallaban igualmente en sectores difusores de conocimiento, como el metalmecánico y el sector TIC (Parra, 2016).

De la segunda fase, que corresponde al PAE y al Fondo de Capital Semilla, se obtuvieron nuevos aprendizajes. El primero fue que no necesariamente el otorgamiento de recursos monetarios daría como resultado una mayor efectividad en la permanencia de los emprendimientos a lo largo del tiempo. Ello llevó, a que en 2015, se tomará la decisión de acabar con el Fondo de Capital Semilla, y se reorientara a un capital de apalancamiento con recursos para fortalecer el acompañamiento en la fase de incubación, dotación de capacidades a los emprendedores y apoyo para mejorar otras actividades, como el networking y la gestión comercial.

Finalmente, en lo que lleva implementado el programa de EAN Impacta se ha logrado aprender que los empresarios, a lo largo de su dinámica empresarial, se enfrentan a problemas ante los cuales, a veces, no se tiene una respuesta. Por lo tanto, es necesario capacitar y orientar a los emprendedores para que asuman la toma de decisiones y persistan en el proceso y no se pierdan los esfuerzos de acompañamiento e incubación. Es necesario innovar en nuevas estrategias de apoyo a las empresas, con sistemas de información para el monitoreo y evaluación de los procesos, y con sistemas expertos de apoyo en la solución de problemas empresariales, para que las estrategias empresariales tengan efectos positivos. 


\section{Referencias bibliográficas}

Ambrose, G. \& Harris, P. (2010). Basics Design: Design Thinking. Laussane: AVA Publishing S.A.

Bhatli, D. (2014). Best Practices at top university business incubators, cases and insights 2014, UBI Index, Benchmarking Incubation Globally.

Cantillon, R., (1896). Ensayo sobre la naturaleza del comercio en general. México: Fondo de Cultura Económica, Ed. 1996.

Foss, Ni. \& Klein, P. (2014). Entrepreneurship and the economic theory of the firm: any gains from trade? CORI Working Paper. 2004-09. Recuperado: http://ssrn. com/abstract=596226or http://dx.doi. org/10.2139/ssrn.596226

Hayek, F., (1934). Razón y evolución. Derecho, legislación y libertad. Normas y Orden. 1. Capítulo 1. Madrid, España: Unión Editorial, Ed. 1994.

IDEO. (2016). Our Approach: Design Thinking. Tomado de: https://www.ideo.com/about/

Kantis, H., Ishida, M. \& Komori, M. (2002). Empresarialidad en economías emergentes: Creación y desarrollo de nuevas en América. Banco Interamericano de Desarrollo (BID), Washington, USA.

Kantis, H., Angelelli, P. \& Moori, V. (2004). Desarrollo emprendedor: América Latina y la experiencia internacional. Banco Interamericano de Desarrollo (BID). Washington, USA.

Kirzner, I., (1998). Competencia y empresarialidad. Madrid: Unión Editorial.

Lederman, D., Messina J., Pienknagura, S., \& Rigolini J., (2014). El emprendimiento en
América Latina: muchas empresas y poca innovación. Washington. Banco Mundial.

Ministerio de Comercio, Industria y Turismo, Asociación Colombiana de Universidades (2014). El estado del arte del emprendimiento universitario. Recuperado de:www.mincit.gov.co/minindustria/ descargar. php?id=71364

Mises, L. V. (2001). La acción humana. Madrid: Unión Editorial.

Parra, L. y Argote, M. (2013). La gestión en el proceso de creación empresarial: el caso de IN3 de la Universidad EAN de Colombia.

Peña-Vinces J., Bravo S., Álvarez F., Pineda D., (2010). Análisis de las características de incubadoras de empresas en Colombia: un estudio de casos. Journal of Economics, Finance and Administrative Science, J. econ. finance adm. sci., 16(30).

Perdomo,G., (2010). Empresarialidad y empresa: una aproximación desde la Escuela Austriaca. Estudios Gerenciales ed: Universidad Icesi, 26 115, pp.161-180, 2010.

Ries, E. (2011). The lean startup: How today's entrepreneurs use continuous innovation to create radically successful businesses. Nueva York: Crown Business.

Reynolds, P., Bygrave, W., Autio, E., Cox L. \& Hay, M. (2001 y 2000). Global Entrepreurship Monitor, Executive Reports Recuperado de: www.entreworld.org

Schumpeter, J. (1934). Entrepreneurship as Innovation. Entrepreneurship: The Social Science View, pp.51-75.2000. Edición (2000) Recuperado de: http://ssrn.com/ abstract $=1512266$ 